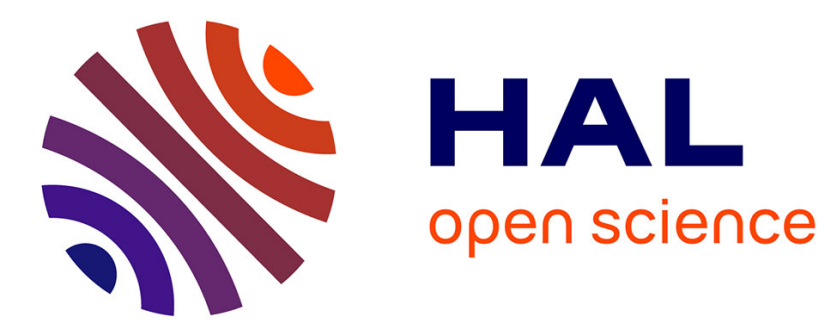

\title{
Self-Healing Distributed Scheduling for End-to-End Delay Optimization in Multihop Wireless Networks with $6 \mathrm{TiSCH}$
}

\author{
Inès Hosni, Fabrice Theoleyre
}

\section{- To cite this version:}

Inès Hosni, Fabrice Theoleyre. Self-Healing Distributed Scheduling for End-to-End Delay Optimization in Multihop Wireless Networks with 6TiSCH. Computer Communications, 2017, 110, pp.103-119. 10.1016/j.comcom.2017.05.014 . hal-02565978

\section{HAL Id: hal-02565978 https://hal.science/hal-02565978}

Submitted on 6 May 2020

HAL is a multi-disciplinary open access archive for the deposit and dissemination of scientific research documents, whether they are published or not. The documents may come from teaching and research institutions in France or abroad, or from public or private research centers.
L'archive ouverte pluridisciplinaire HAL, est destinée au dépôt et à la diffusion de documents scientifiques de niveau recherche, publiés ou non, émanant des établissements d'enseignement et de recherche français ou étrangers, des laboratoires publics ou privés. 


\title{
Self-Healing Distributed Scheduling for End-to-End Delay Optimization in Multihop Wireless Networks with $6 \mathrm{TiSCH}^{\star}$
}

\author{
Inès Hosni ${ }^{\mathrm{a}, *}$, Fabrice Théoleyre ${ }^{\mathrm{b}, *}$ \\ ${ }^{a}$ Laboratory Systems Communications, University of Tunis El Manar, National Engineering School of Tunis, Tunisia \\ ${ }^{b}$ CNRS, ICube Laboratory, University of Strasbourg, Boulevard Sebastien Brant, 67412 Illkirch Cedex, France
}

\begin{abstract}
Time Slotted Channel Hopping (TSCH) is an amendment of the the IEEE 802.15.4 working group to provide a low-power Medium Access Control (MAC) for the Internet of Things (IoT). This standard relies on techniques such as channel hopping and bandwidth reservation to ensure both energy savings and reliable transmissions. Since many applications require low end-to-end delay (e.g. alarms), we propose here a distributed algorithm to schedule the transmissions with a short end-to-end delay. We divide the network in stratums, regrouping all the nodes with the same depth in the DODAG constructed by RPL. Then, different time-frequency blocks are assigned deterministically to each stratum. By appropriately organizing the blocks in the slotframe, we are able to deliver a packet before the end of the slotframe, whatever the route length is. We present a simple analytical study to define the initial size of each block in a homogeneous scenario. We experimentally analyze the behavior of our strategy to validate its ability to provide both high reliability and low latency in a distributed manner.
\end{abstract}

Keywords: IEEE802.15.4-TSCH; end-to-end delay; self-healing; distributed scheduling; autonomous; large-scale experiments

\section{Introduction}

During the last years we have witnessed the emergence of a new paradigm called the nternet of Things (IoT), able to connect the physical and digital worlds. Smart Objects start to be interconnected, enabling new usages for e.g. smart buildings or home automation 2 .

After best-effort solutions, industrial networks now require high reliability with low latency [3]. The IEEE802.15 working group has proposed the IEEE802.15.4-2015 amendment [4] to deal with low power lossy networks. In particular, the TimeSlotted Channel Hopping (TSCH) mode aims to improve the reliability in noisy environments [5]. This standard was designed to cope with the specificities of the Internet of Things, where devices transmit periodically their measures to the Internet, through a border router [6]. A global schedule provides a deterministic medium access: the standard assigns a set of time-frequency blocks to each radio link. By appropriately selecting the set of transmitters in a given channel and timeslot, the network

\footnotetext{
${ }^{\star}$ A short conference version of this paper has already been published [1]

* Corresponding authors

Email addresses: ines.hosni@hotmail.fr (Inès Hosni), theoleyre@unistra.fr (Fabrice Théoleyre)
} 
avoids any intern collision. Channel hopping and over-provisioning help to defeat external interference, making this technology robust.

Nodes maintain a slotframe structure, i.e. a sequence of timeslots which repeats over time. Then, the schedule specifies the action of each node for each timeslot. At the beginning of a slot, a node may either sleep or turn its radio on to receive or transmit a frame.

However, the current stack of protocols for the Internet was not designed initially to cope with a MAC layer based on reservations. Thus, the $6 \mathrm{TiSCH}[7$ working group aims to define a set of protocols to bind the Link and Network layers. While RPL [8] constructs the end to end routes and maintains the control plane, $6 \mathrm{P}[9$ is in charge of reserving the cells (i.e. transmissions opportunities) between a pair of neighbors. The Scheduling Function has finally to decide how many cells should be reserved with each neighbor.

To assign resources for each packet is currently a very challenging objective. We have to define the set of timeslots and channels each pair of nodes uses to exchange packets. Several propositions focused on constructing a centralized schedule [10, 11]. In particular, TASA [12] relies on centralized matching and coloring procedures to assign the timeslots and channels to each radio link.

Tinka et al. [13] proposed rather a distributed approach for mobile ad hoc networks. DETAS [14] computes distributively micro-schedules which are finally merged at the border router. However, these approaches are not reactive, they require deep modifications when e.g. a new flow is injected in the network. Besides, we aim here also to guarantee flow isolation: orthogonal resources should be allocated to each application. In particular, the performance of one flow should be independent of the other ones, i.e. an amount of bandwidth has to be dedicated for each application.

Recently, SF0 [15] describes the standard behavior of the 6TiSCH stack: it estimates the number of transmission opportunities required to transmit all the traffic received and generated by the node itself. It uses an hysteresis function to avoid oscillations. While SF0 computes the number of cells required, we here focus on selecting which cells to use. We adopt consequently a complementary approach.

In this paper, we propose to rely on the current $6 \mathrm{TiSCH}$ stack to provide a fully autonomous and reactive solution. It allocates slots on-demand, using only local information. More precisely, we aim to guarantee a maximum end-to-end delay: the packets must be delivered before the end of the current slotframe, even if some retransmissions are required because some radio links are unreliable.

We propose here a distributed scheduling based on stratums: all the nodes with the same hop distance from the border router form a stratum, and all their transmissions are scheduled in the same time-frequency block (a band). We have to carefully size these blocks to avoid the funneling effect [16. Besides, we propose to re-use the distributed Scheduling Function SF-loc [17] to decide how many cells to allocate. More specifically, a node reserves dynamically new timeslots in the correct band when it has too much traffic to forward. Scheduling all the retransmissions in the same band allows the end-to-end delay to be contained.

To the best of our knowledge, we propose the first method in $6 \mathrm{TiSCH}$ to upper bound the end-to-end delay while assigning reactively the cells (transmission opportunities).

The contribution of this paper is fourfold:

1. we present a distributed scheduling so that a packet is delivered before the end of the current slotframe. Our strategy consists in dividing the network in stratums, and to allocate different timeslots for each stratum to avoid collisions. The blocks are then sequentially organize to guarantee that a packet is relayed during the next contiguous block, i.e. before the end of the slotframe;

2. we present a simple analytical study to define the default size of each block to reduce the funneling effect [16], the amount of cells in a block being proportional to the amount of traffic to forward;

3. we implement this scheduling algorithm in the $6 \mathrm{TiSCH}$ stack while preserving flow isolation;

4. we present experimental results conducted on the large-scale FiT IoT-Lab testbed, highlighting the relevance of the stratum approach. 


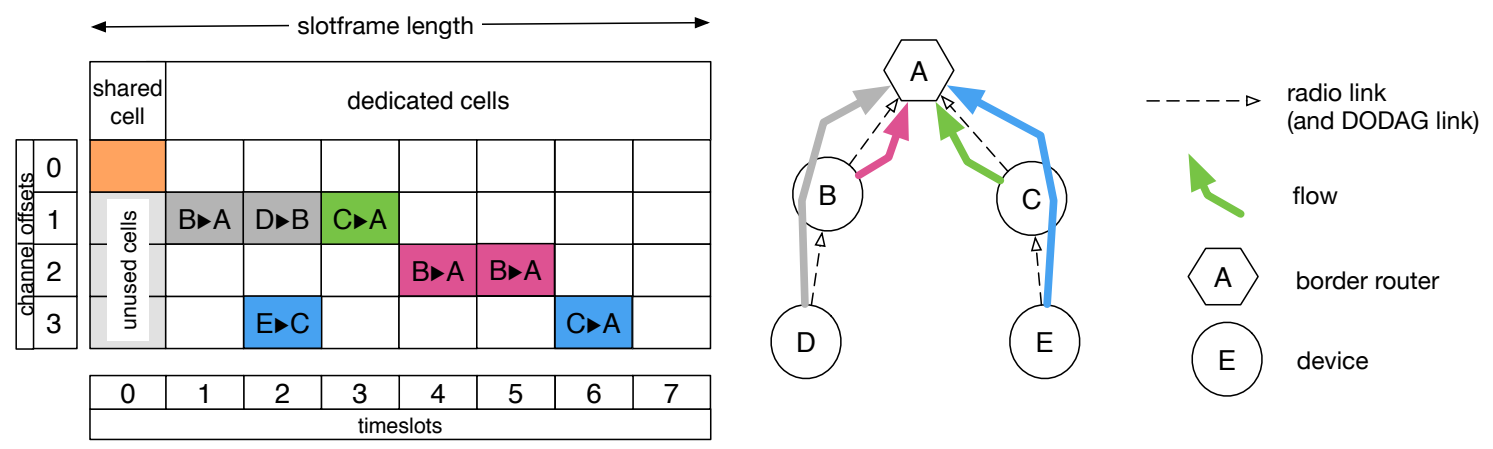

Figure 1: Schedule in a IEEE802.15.4TSCH network - illustration of a slotframe with 8 timeslots

\section{Related Work}

The Industrial Internet of Things has enabled a large set of applications. While traditional wireless sensor network applications have been assumed as delay-tolerant and non sensitive to packet losses, realtime requirements are now of primary importance [18].

Thus, more and more solutions rely on a slow channel hopping mechanism paired with a tight synchronization of the transmissions to both avoid collisions and save energy. A schedule has to be constructed to guarantee a high reliability and a low latency for end-to-end flows. To be efficient, the schedule should carefully select the radio links to schedule simultaneously. We should both avoid collisions because it impacts both the reliability and the energy efficiency, and multiplex the transmissions of non-interfering nodes.

In particular, the capture effect is very common [19]: even if two interfering nodes transmit simultaneously a frame, a receiver may be able to decode the strongest signal. Besides, the capture effect in TDMA networks is high since the network is globally synchronized and deterministic The capture effect represents a great opportunity to increase spatially channel re-use [20].

\subsection{IEEE802.15.4-TSCH}

IEEE802.15.4-2015 has proposed the TSCH mode for industrial wireless sensor networks. To improve the reliability while maximizing energy savings, a schedule may be computed by the Path Computation Element (PCE) and distributed to all the nodes. The schedule is contained in a slotframe (8 timeslots and 4 channels in Figure 1), and this slotframe is repeated periodically. The Absolute Sequence Number (ASN) counts the number of timeslots since the beginning, so that all the nodes have a common time reference.

At the beginning of each timeslot, a device examines the schedule to know if it has to wake-up to transmit or receive a packet. A timeslot can be either dedicated (without contention) or shared (with a slotted CSMA-CA mechanism to solve the conflicts between the contenders).

A cell is defined by a pair of timeslot and channel offset. During an incoming cell, a node waits for a reception, while it is in transmission mode during the outgoing cell. When a node is neither receiver nor transmitter, it turns its radio off.

To improve the reliability, TSCH proposes to implement slow channel hopping. In the TSCH jargon, a cell is a pair of timeslot and channel offset. The channel offset is translated into a physical frequency at the beginning of the timeslot:

$$
f r e q=f\left(\left(A S N+c h_{o f f s e t}\right) \% n_{c h}\right)
$$

where ASN is the absolute sequence number of the timeslot, $c h_{\text {of } f \text { set }}$ is the channel offset assigned to this cell in the schedule, $\%$ the modulo operator, $n_{c h}$ the number of physical channels, and $f()$ is a mapping function, spreading the load on all the physical channels. 
Let's consider the schedule illustrated in figure 1. As recommended by 6TiSCH minimal 21, we have one shared cell at the beginning of the slotframe, using the channel offset 0 . The shared cell is typically used for control traffic (i.e. new reservations, control packets for routing, beacons, etc.). The other cells are dedicated: only the owner of the cell can transmit a packet, without contention. Each DODAG link has one or several cells to forward the packets. The link $A \rightarrow \mathrm{B}$ (pink) reserved for instance two dedicated cells because it has many packets to forward, or because retransmissions are expected.

\section{2. $6 \mathrm{TiSCH}$}

The 6TiSCH IETF working group aims to define the protocols to operate IPv6 (6LoWPAN) over a reservation-based MAC layer (IEEE802.15.4-TSCH). 6TiSCH introduces the concept of tracks to reserve an amount of dedicated cells for a particular flow 22. Hop by hop, each intermediary node inserts in its schedule some cells for each non best effort flow (i.e. track instance $\neq 0$ ). Label switching may be implicit: a node knows the track associated to an incoming cell, extracted directly from the schedule. Thus, it just has to forward this packet in an outgoing cell with the same track id.

The protocol $6 \mathrm{P}$ defines how a node may negotiate a cell with a neighbor: the enquirer specifies a list of available $<$ timeslot, channel_offset $>$ and the number of cells it asks for [9]. The neighbor will accept the request if these cells are available also in its schedule: it sends an Information Element to notify the enquirer. However, it does not define which cells should be selected.

Let's consider the figure 1 which illustrates a possible schedule with 4 different tracks. We see that the link $B \rightarrow A$ supports two tracks with respectively the source $B$ (in pink) and the source $D$ (in gray). Since each track uses a different set of cells, flows are isolated: the packets of $D$ do not impact the traffic of $B$.

Besides, the incoming cell in $B$ for the flow from $D$ (in gray) is scheduled after the outgoing cell. This means the node $B$ has to buffer the packets during a long time, until the next slotframe. Thus, the end-toend delay is quite large. Thus, we have to carefully schedule the slots for each track to limit this buffering delay. In this paper, we use the $6 \mathrm{TiSCH}$ stack to reserve reactively the cells for each track: each flow reserves hop-by-bop its own dedicated cells.

6TiSCH makes a clear distinction between how the cells are negotiated (using the protocol 6P [9]), and how many cells have to be reserved or released (using a Scheduling Function).

\subsection{Traffic Aware Scheduling Algorithm}

To assign the resources for each packet is currently a very challenging objective: $6 \mathrm{P}$ has to define which cells should be used for each flow, along each hop of the route to the border router. While 6 TiSCH defines how the cells are negotiated (with the $6 \mathrm{P}$ protocol), any scheduling algorithm may be actually implemented.

\subsubsection{Centralized Approaches}

Tsitsiklis et al. [11] study the tradeoff between a centralized and a distributed scheduling. By adopting a queue theory based approach, they demonstrated a centralized approach is more efficient. Ghosh et al. [23] propose to minimize the schedule length in a multichannel TDMA environment. However, the authors do not consider packet losses.

Yan et al. 24 construct an optimal schedule for time-sensitive flows: new cells are inserted in the schedule if the end-to-end reliability is insufficient until the deadline constraint is not fulfilled. TASA propose to construct a compact schedule for a multihop IEEE802.15.4TSCH network [12: the same slotframe may be repeated more frequently to increase the network capacity. Yigit et al. 25] study the impact of routing on the schedule: using unreliable links increases the number of timeslots required to achieve a minimum reliability. Dobslaw et al. 26 propose to reserve additional timeslots for retransmissions. Modesa 27 exploits a linear programming formulation to minimize the size of the schedule. In particular, the authors explore the interest of using a multi-interface sink to improve the network capacity.

Phung et al. 28, propose to use a Reinforcement Learning based scheduling algorithm to cope with a variable traffic. However, the authors do not propose to use dedicated cells: the nodes have always to execute a CSMA-CA phase before transmitting their packets. 
Lee et al. 29 address the reliability problem: if a given radio link is broken, it impacts all the flows forwarded through this link. Thus, the authors propose to exploit substitute paths, so that the packets keep on being forwarded through alternative radio links, using backup cells. Alternatively, Opportunistic scheduling explores further this direction, by enabling anycast at the link layer. Each node can use a single transmission to send a packet to all its next hops: the first to acknowledge will be in charge of relaying the packet. This technique helps to improve the reliability when one of the next hops becomes faulty [30].

All these centralized algorithms have been evaluated with a numerical analysis in $\mathrm{C}$ (24]), Python ([14, 12]), Matlab ([25]), or Octave ([31]). The most complex models use a Rayleigh fading to simulate radio links with different ETX values. In particular, we need to know a priori which radio links exist in the topology, their link quality, the amount of packets generated by each node, etc. Such information is very complex to collect in many practical situations, and inconsistencies in the schedule may quickly arise if the conditions change. Consequently, these approaches are well suited for industrial networks in controlled environments with strict requirements on reliability and delay, where everything is known pre-deployment.

\subsubsection{Hierarchical Approaches}

DeTAS propose a decentralized version of TASA [14: the children of the border routers collect the radio topology of their subtree to compute independently the schedule of their descendants (called microschedule). Finally, the micro-schedules are re-arranged into a globally acceptable schedule. Thus, the schedule computation is still concentrated in a few nodes, which are aware of the radio interference.

Wave [31] constructs a schedule such that a packet is delivered before the end of the slotframe, even if it has to be relayed by intermediate nodes. The wave is in charge of scheduling the $i^{\text {th }}$ transmission of each node. However, the authors do not describe the signaling mechanisms to change the schedule on-thefly, and to take into account the traffic requests of each node at the beginning of a slotframe. Restarting the scheduling process at the beginning of each slotframe would be particularly expensive. In this paper, we adopt the same objective, i.e. delivering the packet before the end of the slotframe, but we adopt a distributed approach.

\subsubsection{Distributed Approaches}

Orchestra was recently proposed 32 to construct a TSCH schedule in a distributed manner. A real performance evaluation in a testbed proves the ability of Orchestra to set-up efficiently a distributed schedule. However, the focus was not given on minimizing the end-to-end delay. Besides, the number of cells for each radio link is fixed, whatever the quantity of traffic a node has to forward to its parent. Finally, Orchestra does not use the $6 \mathrm{TiSCH}$ tracks, and does not guarantee flow isolation.

SF0 [15] represents the default behavior of $6 \mathrm{TiSCH}$ : a node monitors the amount of traffic it has to forward and it generates. The number of cells in its schedule must be at least equal to the amount of packets to transmit (i.e. received and generated packets). Dujovne et al. propose to over-provision a fixed number of cells toward each neighbor to react to changes. Besides, an hysteresis approach is also implemented for the cell allocation to avoid over-reacting to transient changes. SF0 also provides a relocation policy: when the PDR of a given cell is significantly below the average PDR, the corresponding cell is moved in the schedule, i.e. a collision probably occurs. Recently, Domingo-Prieto et al. 33 propose to adopt a Proportional, Integral, and Derivative approach, inspired from the control world, to accelerate the convergence while limiting the number of reconfigurations.

DISCA proposes a lock-based scheduling approach [34]. Each node is assigned a priority, according to the quantity of traffic they forward. The algorithm proceeds iteratively, allocating in the step $i$ a slot to the $i^{t h}$ transmission of each node. The transmitter notifies its interfering neighbors of the cell is selects so that it is locked in the neighborhood.

Duy et al. 35] propose to minimize the number of collisions in the schedule by dividing the slotframe in portions of equal length. Then, each source node selects the portion which contains the lowest number of already scheduled cells. Thus, each node has to monitor the occupancy ratio of each portion.

Chang et al. 36] proposed a Scheduling Function to minimize the end-to-end delay. For this purpose, the transmitting cell is allocated as close as possible after the receiving cell, in order to reduce the buffering delay. However, the authors do not consider packet losses: only one cell is reserved, whatever the link quality 
is. We propose here to tackle this problem.

\section{Problem Statement}

We consider a Low Power Lossy network (LLN) organized by RPL in a single DODAG (Destination Oriented Directed Acyclic Graph), anchored in a border router. Each node maintains its rank denoting its virtual distance from the border router. Typically, the rank may be the average cumulative number of transmissions (ETX) along the path to the border router [8].

We consider here the standard version of RPL, where a node uses only its preferred parent to route its packets. Thus, a node has to negotiate a set of cells with its parent, next hop to the border router. The nodes may send their data packets at any periodicity. We use tracks to reserve some dedicated cells for each flow with traffic isolation.

To maintain a steady state, a node should have enough cells to transmit all the received packets. More precisely, all the packets present in the buffer at the beginning of the slotframe have to be delivered before the end of the same slotframe. Thus, a node should have always enough cells to handle the worst case, even when retransmissions are required.

\subsection{The Limits of a Centralized Scheduling}

Several algorithms assign the timeslots and the channel offsets in a centralized manner. However, centralized scheduling faces to several challenges:

Radio topology: the scheduler needs to know the list of neighbors for each node in the network. This list is used to decide which routes have to be used. For instance, TASA [12] assumes RPL is used to create a DODAG, and the RPL routes are then used by the controller. However, the signaling mechanism to continuously monitor the neighbors, and to push the modifications to the controller are not described;

Estimating interference: since the scheduling is centralized, colliding cells would be very prejudicial. Indeed, these cells will waste energy and bandwidth, and a new global schedule has to be recomputed;

Reliability: scheduling algorithms often implicitly assume perfectly reliable links (no packet is lost), such as TASA does [12]. Practically, the packet delivery ratio may not be $100 \%$, as we measured on our testbeds. While Schedex [26] addresses this reliability problem, traffic isolation is not considered;

Efficient distribution of the schedules: the Path Computation Element (PCE) is the central entity to compute the schedule. Then, the local schedules have to be pushed in every node, using for instance CoAP. The amount of control packets generated by such configuration is significant. Besides, dealing with inconsistencies (some nodes may listen to the incorrect schedule because the reconfiguration failed) may represent a challenge;

Self-healing: the link quality or the traffic may be time-variant. A small change requires to change globally the schedule and to reconfigure the whole network. This has a very negative impact on the energy consumption, and on the performance during the reconfiguration phase.

To the best of our knowledge, no centralized scheduling was evaluated experimentally so far. For instance, the numerical analysis were conducted in C ([24]), Python ([14, 12]), Matlab ([25]), Octave ([31]). While the most complex models take into account the radio link quality, most centralized scheduling algorithm do not focus on collecting the control information, change dynamically the schedule, etc.

For all these reasons, we propose here a localized scheduling, which doesn't assume any specific condition. The schedule is updated on-the-fly to deal with interference, radio topology or traffic variations.

\subsection{Large end-to-end Delay with a Random Distributed Scheduling}

We first compute here the average end-to-end delay we may obtain with a random scheduling algorithm. More precisely, when a pair of nodes has to negotiate a cell, the transmitter selects randomly a free timeslot and channel offset (algo 1). Then, it sends a request to the receiver to verify if this cell is also free for it. The process reiterates until a common free cell is selected. 


\begin{tabular}{|c|c|}
\hline $\operatorname{ETX}(A, B)$ & ETX value from $A$ to $B$ \\
\hline$n b C e l l s O u t(A, B)$ & Number of outgoing cells (transmissions) from \\
\hline$n_{\text {cell }}$ & $\begin{array}{l}\text { slotframe length (number of cells in the slot- } \\
\text { frame) }\end{array}$ \\
\hline$n_{c h}$ & Number of channels \\
\hline $\begin{array}{r}T_{\text {slot }} \\
p=\{S . . D\}\end{array}$ & $\begin{array}{l}\text { Timeslot duration (by default 10ms) } \\
\text { path from } S \text { to } D\end{array}$ \\
\hline$W_{\text {ring }}$ & Euclidean width of a ring \\
\hline $\mathcal{R}_{n w}$ & $\begin{array}{l}\text { Euclidean radius of the network (distance of } \\
\text { the node farthest from the border router) }\end{array}$ \\
\hline $\mathcal{T}$ & Quantity of traffic generated by each node \\
\hline $\mathcal{B}=\left\{B_{i}\right\}_{i \in[1 . .|B|]}$ & $\begin{array}{l}\text { Set of Blocks for the stratum scheduling algo- } \\
\text { rithm }\end{array}$ \\
\hline $\operatorname{size}\left(B_{k}\right)$ & Size (= number of cells) of the block $B_{k}$ \\
\hline$d_{\max }$ & $\begin{array}{l}\text { Maximum hop distance for block re-utilization } \\
\text { (i.e. frequency reuse) }\end{array}$ \\
\hline
\end{tabular}

Table 1: Notations

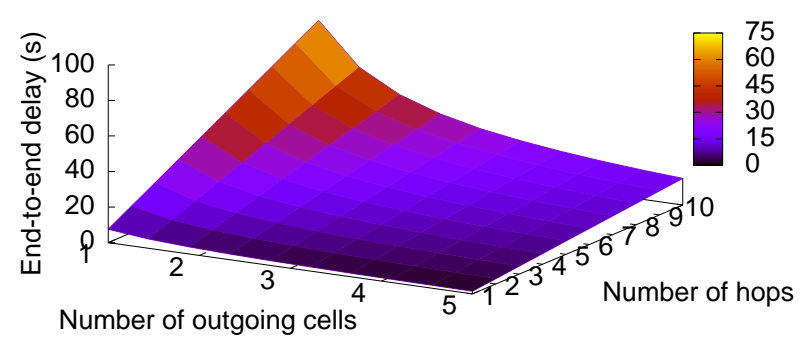

Figure 2: End-to-end delay with a slotframe of 1001 timeslots (=10s) including 1 shared slot, with perfect radio links (ETX=1)

Let's consider that a packet is enqueued at the beginning of the slotframe. A packet has to be enqueued until the next outgoing cell, and these cells are uniformly distributed in the slotframe. Thus, it needs to be buffered on average during:

$$
\text { OutCell_delay }=\frac{n_{\text {cell }} * T_{\text {slot }}}{2 * n b C e l l s O u t(A, B)}
$$

with $n_{\text {cell }}$ being the slotframe length, $T_{\text {slot }}$ the duration of a timeslot and $n b C e l l s O u t(A, B)$ the number of outgoing cells.

Besides, a packet needs possibly several retransmissions if the link is unreliable. Precisely, it needs on average ETX transmissions [37, i.e. the average number of transmissions from A to B before receiving an acknowledgement.

Finally, the packet is enqueued during:

$$
\operatorname{hop} \_d e l a y(A \rightarrow B)=\frac{n_{\text {cell }} * T_{\text {slot }}}{2 * n b \operatorname{CellsOut}(A, B)} * \operatorname{ETX}(A, B)
$$

with $\operatorname{ETX}(A, B)$ the ETX from A to B.

Since we assume a packet may be generated at anytime, the end-to-end delay for a packet along the 

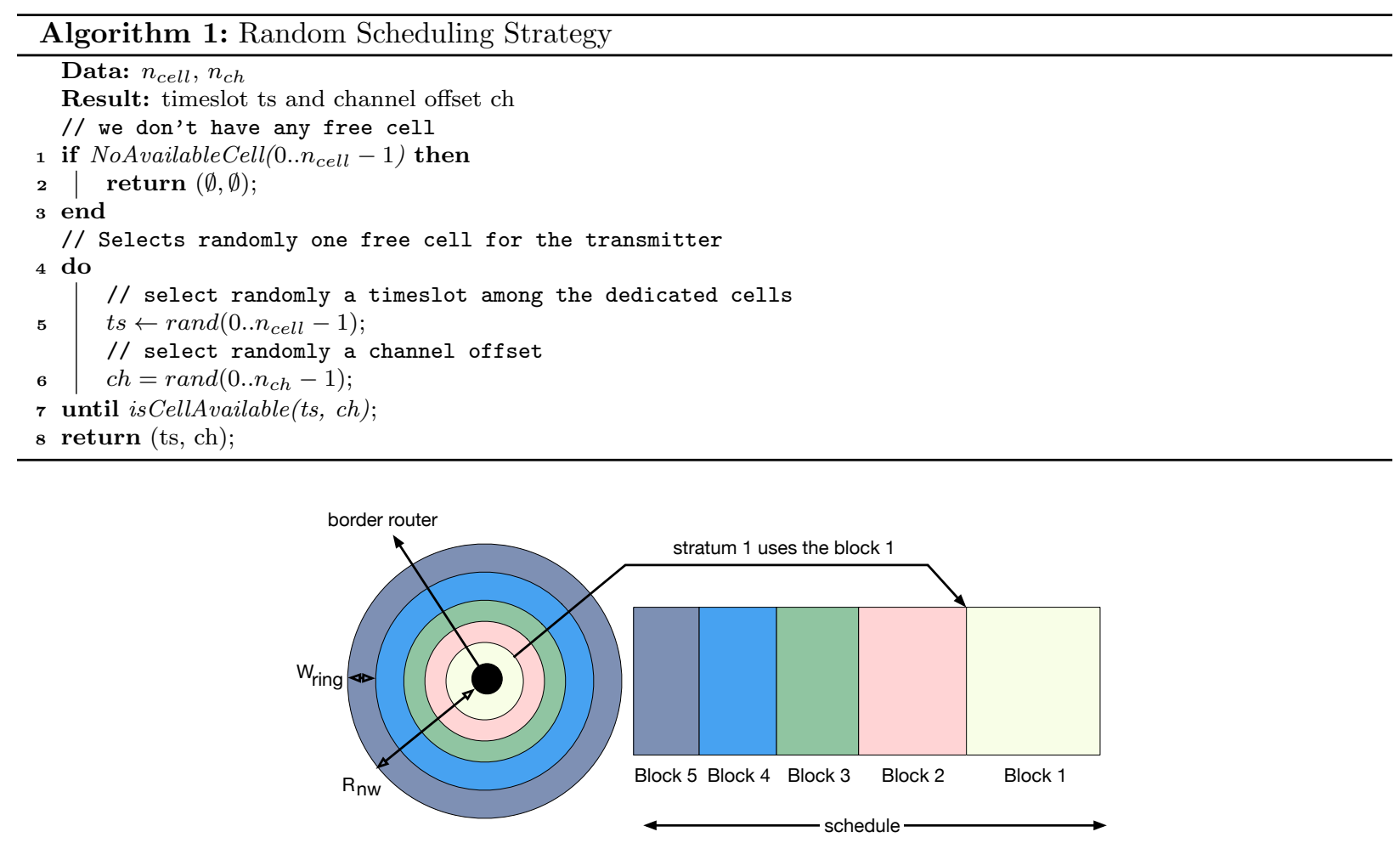

Figure 3: Strategy of the Stratum Scheduling

route $p$ is finally:

$$
\operatorname{E2E\_ Delay}(p)=n_{\text {cell }} * T_{\text {slot }} \sum_{i=1}^{|p|-1} \frac{\operatorname{ETX}\left(N_{i}, N_{i+1}\right)}{2 * \operatorname{nbCellsOut}\left(N_{i}, N_{i+1}\right)}
$$

with $p=\left\{\left(N_{i}, N_{i+1}\right)\right\}$ being the path from A to B.

Figure 2 illustrates the end-to-end delay obtained with a typical slotframe of 1001 slots. This slotframe models typically an application where a flow has to send one packet every 10 seconds.

In conclusion, we may reduce the end-to-end delay by allocating more cells. However, this overprovisioning increases both the number of collisions and the energy consumption.

Besides, the end-to-end delay increases linearly with the network diameter. While we implemented this solution as a comparison purpose, we have to propose a specific smarter solution to reduce this end-to-end delay.

\section{Distributed Stratum Scheduling}

We propose here a localized scheduling to be robust to any change (topology, traffic, routing paths). We are convinced a distributed schedule, reactively computed, may cover the needs of various applications (e.g. smart homes). We propose to guarantee a maximum end-to-end delay: any packet which is enqueued at the beginning of the slotframe should be delivered before the next slotframe. Thus, by fixing the slotframe length, the network administrator is able to fix also the maximum tolerable delay.

We divide the network in stratums: all the nodes which are $k$ hops far from the border router constitute the stratum $k$ (Fig. 3). We denote by depth the hop distance from the border router. Thus, each node includes in its DIO (DODAG Information Object, a RPL control packet) a field denoting its depth (which is also its stratum number). 
We can note that we don't use minimum hop routing: the depth metric is uniquely used by our scheduling algorithm, and RPL may use any routing metric. In other words, the rank and the depth are different metrics. The depth is computed as the depth of the preferred parent already selected by RPL (according to any routing metric), increased by one.

We have now to assign a time-frequency block (a band) to each stratum. All the nodes in the stratum $k$ must reserve a timeslot from the block $k$. We construct a global schedule in which the blocks from contiguous stratums are consecutive. This way, we guarantee a packet is delivered before the end of the slotframe.

We adopt here the Scheduling Function SFloc proposed in [17]: each node adapts locally and dynamically the number of required cells for each track. Besides, in the stratum strategy, a node picks a cell from the block assigned to its stratum. Since the stratum is directly determined by the depth, a node implements a localized scheduling strategy. However, any localized Scheduling Function (such as SF0) would be here accurate.

We have now to define which block will be assigned to each stratum. We will first focus on the upload case (e.g. periodical measures are pushed to a border router), and we will explain further how to adapt our solution to cope also with the inverse direction.

\subsection{Defining the size of each stratum}

We have to define which portion of the schedule is assigned to each block. We propose here to consider an ideal case, where the nodes are uniformly distributed in a given area, and generate the same amount of traffic. This ideal situation helps us to define which values to use to bootstrap the network. In section 4.3 . we will detail how the network may change on-the-fly these values, to deal with particular situations (e.g. heterogeneous densities, traffic or topologies).

We denote by block all the nodes which are equidistant (in hops) from the border router. We will first explain how we assign a schedule to the nodes which are at most $d_{\text {max }}$ hops far from the border router. We will explain in the next subsection which blocks have to be used for the nodes located farther, and how the value of $d_{\max }$ should be selected.

If we consider each node generates the same amount of traffic, we should not divide the whole schedule in $d_{\max }$ blocks with an equal size: the nodes close to the border router have more traffic to forward. More precisely, on average, a node in the stratum $k$ forwards the traffic of all the nodes from the stratums with a larger depth.

If the deployment is sufficiently dense and all the nodes have the same transmission power, we may assume all the rings have a fixed width $W_{\text {ring }}$ 38. When using ETX as a metric, $W_{\text {ring }}$ is typically the distance at which the packet delivery ratio is almost perfect (i.e. $100 \%$ ). Let $\mathcal{R}_{n w}$ denote the euclidean distance of the node farthest from the border router (i.e. the network radius) (cf. Fig. 3 )

If we assume each node generates the same amount of traffic denoted $\mathcal{T}$, the traffic generated by the stratum is directly proportional to the area of the corresponding ring. Thus, the traffic generated by the stratum $k$ is:

$$
\begin{aligned}
\mathcal{T}_{\text {gen }}\left(B_{k}\right) & =\mathcal{T} * \pi\left(\left(W_{\text {ring }} * k\right)^{2}-\left(W_{\text {ring }} *(k-1)\right)^{2}\right) \\
& =\mathcal{T} * \pi W_{\text {ring }}^{2} *\left(k^{2}-(k-1)^{2}\right)
\end{aligned}
$$

Inside a given stratum, we have frequency re-use: nodes which are sufficiently far may re-use the same cell without colliding. Unfortunately, frequency re-use is less important for stratums closer from the sink: the interfering area spans the whole stratum. Estimating precisely the impact of frequency re-use would require to define formally the ratio of the area of the ring, and of the interfering region.

Let's consider the topology illustrated in Fig. 4. The shaded area in stratum 2 corresponds to the interfering region around the node $A$ : nodes of the stratum 2 but outside this area may re-use the same cells as $A$. In conclusion, the ratio of traffic that a node in stratum 2 has to forward compared with the traffic of a node in stratum 3 should be the ratio of the two corresponding shaded area. 


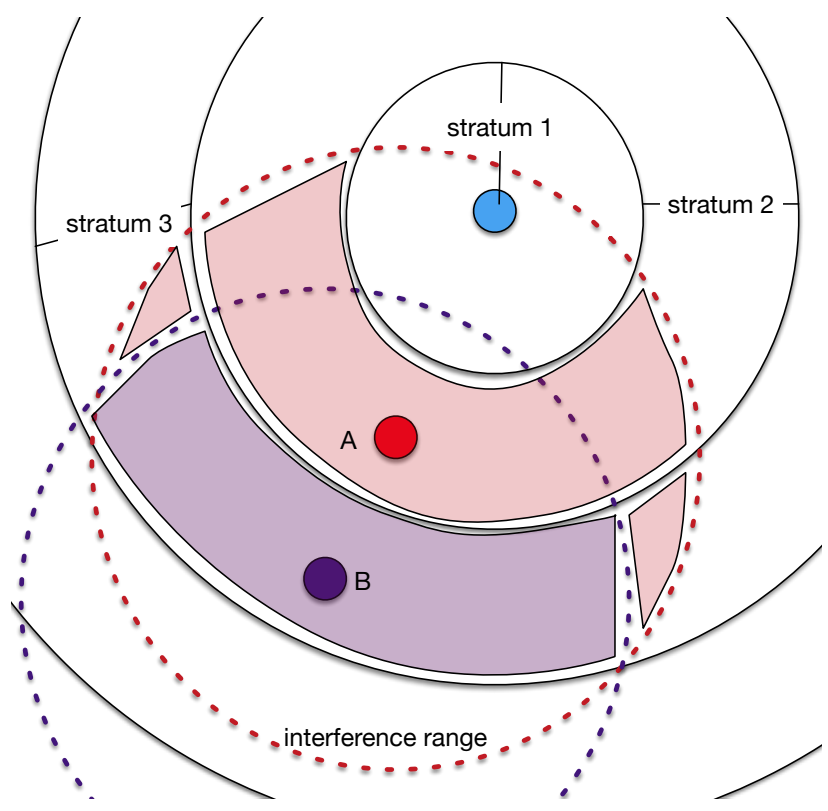

Figure 4: Interference region for the different stratums

However, estimating analytically the size of each area depends on the stratum number, the interference range, etc. Thus, we propose here to over-estimate the level of interference, and to consider that the ratio of traffic the nodes from adjacent stratums have to forward, is equal to the ratio of the corresponding ring areas.

Estimating more precisely the shaded region is expensive. Moreover, this value would only be used initially: the actual block size may be changed later dynamically (cf. section 4.3 below). Thus, we consider our approximation seems reasonable in this situation.

In conclusion, we consider that the block $k$ has to forward the traffic from all the other blocks with a larger depth (and the traffic generated by the nodes of the block $k$ ). In other words, it consists of all the traffic generated in the network, except the rings closest from the border router:

$$
\begin{aligned}
\forall k \geq 1, \mathcal{T}_{\text {fw }}\left(B_{k}\right) & =\left(\pi * \mathcal{R}_{\text {nw }}^{2} * \mathcal{T}\right)-\sum_{i=0}^{k-1} \mathcal{T}_{\text {gen }}\left(B_{i}\right) \\
& =(\pi * \mathcal{T}) *\left(\mathcal{R}_{n w}^{2}-W_{\text {ring }}^{2} * \sum_{i=0}^{k-1}\left(i^{2}-(i-1)^{2}\right)\right)
\end{aligned}
$$

Thus, the size of the block allocated to a ring should be proportional to the amount of traffic it forwards:

$$
\begin{aligned}
\frac{\operatorname{size}\left(B_{k}\right)}{\operatorname{size}\left(B_{0}\right)} & =\frac{\mathcal{T}_{f w}\left(B_{k}\right)}{\pi * \mathcal{R}_{n w}^{2} * \mathcal{T}} \\
\operatorname{size}\left(B_{k}\right) & =\operatorname{size}\left(B_{0}\right) * \frac{(\pi * \mathcal{T}) *\left(\mathcal{R}_{n w}^{2}-W_{r i n g}^{2} * \sum_{i=0}^{k-1}\left(i^{2}-(i-1)^{2}\right)\right)}{\pi * \mathcal{R}_{n w}^{2} * \mathcal{T}} \\
& \left.=\operatorname{size}\left(B_{0}\right) *\left(1-\left(\frac{W_{\text {ring }}}{\mathcal{R}_{n w}}\right)^{2} * \sum_{i=0}^{k-1}\left(i^{2}-(i-1)^{2}\right)\right)\right)
\end{aligned}
$$




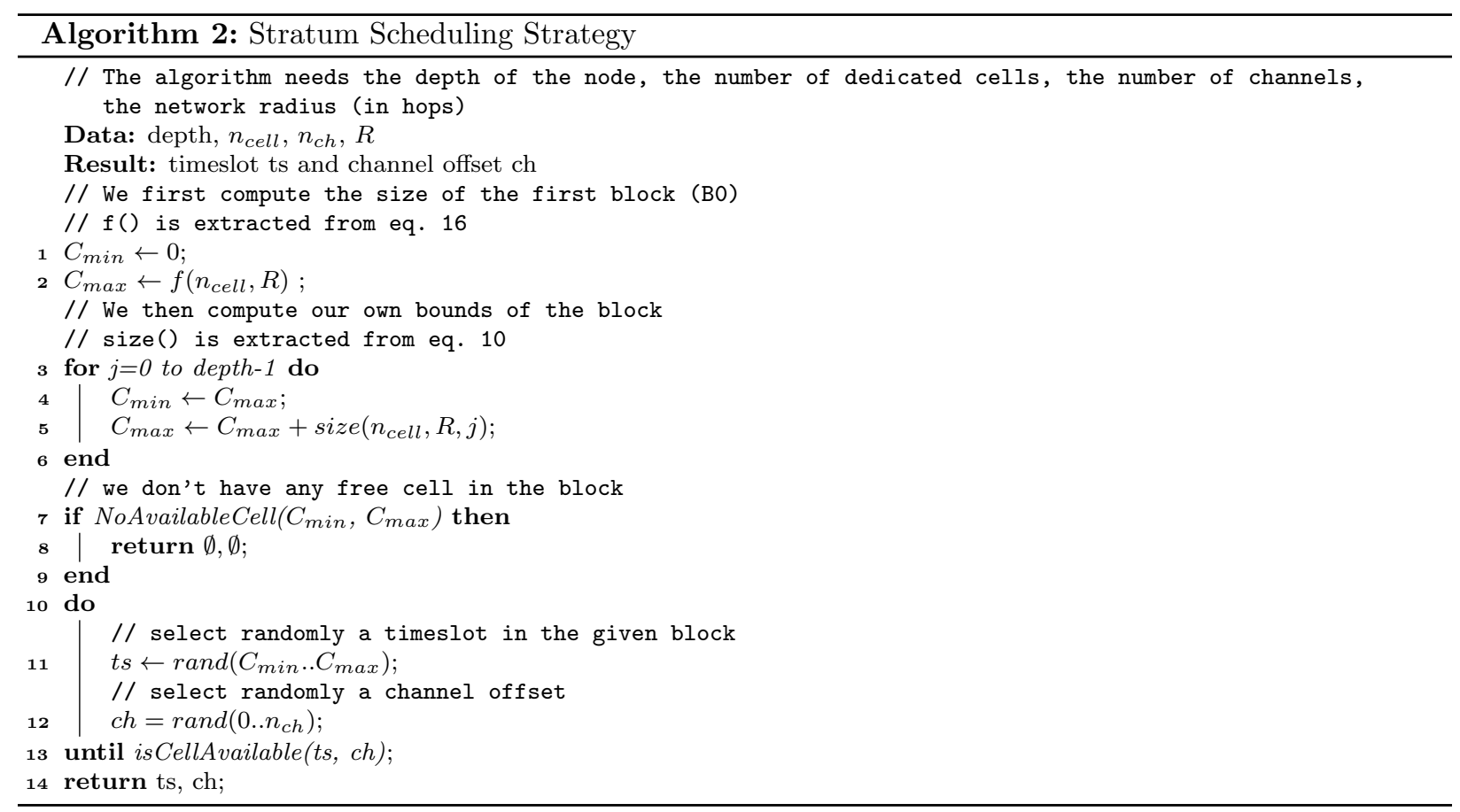

And finally, the union of all the blocks represents the scheduling matrix:

$$
\sum_{i=0}^{|\mathcal{B}|-1} \operatorname{size}\left(B_{i}\right) \leq n_{\text {cell }}
$$

We can reformulate this constraint using eq. 10 to determine size $\left(B_{0}\right)$ :

$$
\operatorname{size}\left(B_{0}\right) * \sum_{i=0}^{|\mathcal{B}|-1}\left(\frac{\mathcal{T}_{f w}\left(B_{i}\right)}{\pi * \mathcal{R}_{n w}^{2} * \mathcal{T}}\right) \leq n_{\text {cell }}
$$

and:

$$
\begin{aligned}
\operatorname{size}\left(B_{0}\right) & =\left\lfloor\frac{n_{\text {cell }}}{\pi * \mathcal{T} * \mathcal{R}_{n w}^{2}} *(\pi * \mathcal{T}) \sum_{i=0}^{|\mathcal{B}|-1}\left(\mathcal{R}_{n w}^{2}-W_{\text {ring }}^{2} * \sum_{j=0}^{i-1}\left(j^{2}-(j-1)^{2}\right)\right)\right\rfloor \\
& =\left\lfloor n_{\text {cell }} \sum_{i=0}^{|\mathcal{B}|-1}\left(1-\left(\frac{W_{\text {ring }}}{\mathcal{R}_{n w}}\right)^{2} * \sum_{j=0}^{i-1}\left(j^{2}-(j-1)^{2}\right)\right)\right\rfloor
\end{aligned}
$$

Algorithm 2 presents the formal definition of our stratum scheduling. A node extracts its stratum and computes recursively with eq. 16 the bounds of the corresponding block. Then, it picks randomly a timeslot in this block, and a random channel offset.

\subsection{Defining the number of stratums}

Many models consider that interference may be neglected when two nodes are sufficiently far [39]. Let's denote by $d_{\max }$ the number of hops after which we may assume no interference arises.

Practically, we fix the number of blocks to be equal to $d_{\text {max }}$. A node in the stratum $k$ will use the block $k \bmod \left(d_{\max }\right)$, where $\bmod ()$ denotes the modulo operator. Thus, nodes which are more than $d_{\max }$ hops 
far from the border router re-use an already allocated cell, without creating interference since we consider they are sufficiently far from each other.

The blocks are also sufficiently large. Indeed, the stratum $\left(d_{\max }-1\right)$ has the smallest block in the network. Thus, all the other stratum larger than $d_{\max }$ have a block which is at least as large. Since the stratum $k$ has always less traffic to forward than a stratum $j(j<k)$, these stratums have enough bandwidth.

\subsection{Updating the blocks size}

Our theoretical analysis relies on a uniform distribution of the nodes, with the same quantity of traffic for each source, etc. Unfortunately, considering realistic conditions would also impact the size of the different blocks. The size of each block can be pre-computed pre-deployment, if the location and the volume of traffic is known a priori, counting the ratio of traffic in each interfering stratum. Since these conditions may be dynamic or cannot be estimated enough accurately, we propose here a dynamic method.

The border router should be able to trigger a reconfiguration, pushing to all the nodes the size of each block. We propose to piggyback in the Enhanced Beacons an Information Element which includes:

- the number of stratums (NB) (4bits): we can safely assume that the same frequency may be reused after 16 hops, which corresponds to a very large interfering range;

- a table of the block's weights $(w(*))$ : the weight defines the ratio of the scheduling matrix associated with the corresponding block. More precisely, the table contains a list of values $w(i)$, denoting the weight $2^{w(i)}$ associated with the block $i$. Then, the block size of each stratum is computed such that:

$$
\sum_{i=0}^{N B-1} 2^{w(i)}=n_{\text {cell }}
$$

If we reserve 4 bits for each weight, the largest block may be 128 larger than the smallest one.

In conclusion, 8 stratums would typically be encoded in 36 bits $\left(4+8^{*} 4\right)$, which seems a very reasonable overhead.

When the configuration changes, a node may have cells in the wrong blocks. Thus, these nodes remove silently the concerned cells: both the transmitter and the receiver use the same stratum's value and they take a consistent decision. Then, the nodes will keep on executing SFloc to renegotiate new cells in the correct blocks if the bandwidth is not anymore sufficient to forward all the queued packets.

The nodes should switch simultaneously to the new configuration. Thus, we propose to piggyback in the EB the minimum time $\left(t_{A S N}\right)$ at which this configuration has to be considered valid. The border router has to push the new configuration sufficiently in advance to cope with retransmissions and packet losses. Typically, the border router should start transmitting the new configuration several EB periods in advance.

\subsection{Up and Download Directions}

While we described only our stratum scheduling for the upload direction - common for wireless sensor networks-, we are also able with this approach to minimize the delay in both directions. The allocation in the download direction has to begin with the first timeslots of the scheduling matrix, and the blocks are organized consecutively.

We consider here the two following situations:

Broadcast (flooding): a packet forwarded by the sink has to be delivered to all the nodes. A node has to reserve one cell to forward a packet to all its children. Similarly, we can guarantee the packet is delivered before the end of the slotframe if each node picks a cell in the correct block.

Unicast: the sink has to send a data packet to a particular node, e.g. a command to an actuator, a reconfiguration to a sensor. One cell has to be selected for each hop in the path, to guarantee the packet is delivered before the end of the slotframe. Thus, our stratum strategy has to organize the allocation along this path. 


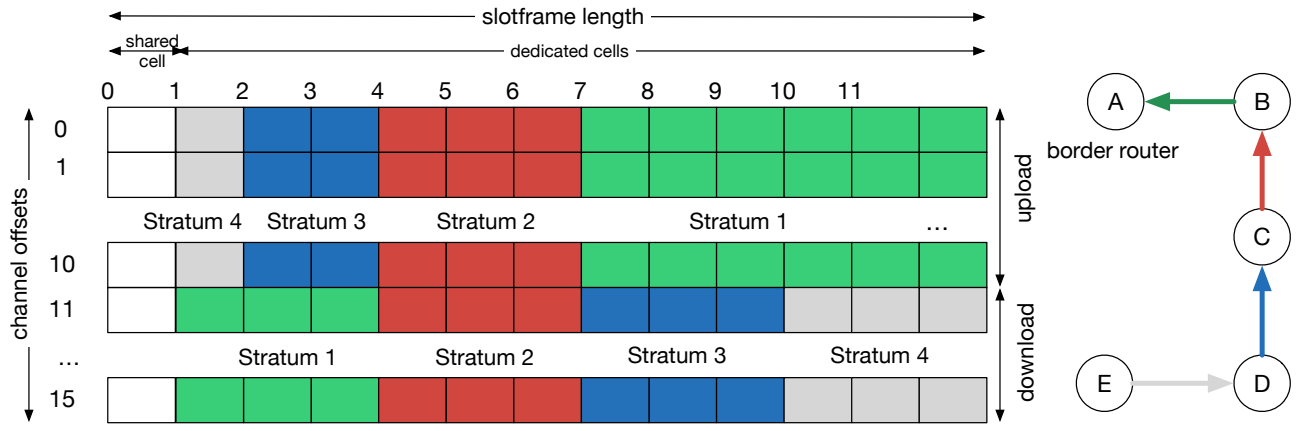

Figure 5: Down and Upload directions for Stratum Scheduling

\subsubsection{Broadcast}

For flooding, we have to reserve one cell for each node in each stratum. Thus, all the nodes have the same amount of flooding cells, wherever they are located in the topology. In conclusion, the size of all the flooding blocks are equal.

\subsubsection{Unicast}

If the unicast flow is sufficiently large, one or several cells have to be reserved during each slotframe, and will be used for the transmission. Thus, the download is in this case handled similarly as the upload traffic.

A more common scenario implies a low, event-triggered upload traffic (e.g. a reconfiguration). To upper bound the end-to-end delay, at least one cell must be reserved for each hop of the path, but most of them are unused, and waste energy. We propose to consequently multiplex different flows in the same track. A node can reserve a cell to forward the packets for different flows to different children. One transmitter and several receivers are associated with this cell, and no collision can occur.

Since a cell is re-used for several receivers, the equation 9 does not hold anymore. Defining the optimal block size depends on the traffic pattern and the inter-packet time. We let this study to a future work.

We keep on relying on the Scheduling Function to decide how many cells have to be reserved. Typically, SFloc counts the number of packets in the queue, and maintains the number of cells at least equal. Then, we force the cells to be picked in the correct block, depending on the stratum of the transmitter. Thus, the upload and download directions are handled in a similar way.

Let's consider the example depicted in figure 5 , where we have twice more upload than download traffic. Thus, the channel offsets 0 to 10 are reserved for upload, while the channel offsets 11 to 15 are reserved for download. In our example all the download blocks have the same size. Besides, the blocks are inversely organized in the schedule to reduce the end-to-end delay.

\section{Self-healing Mechanisms to detect and solve collisions}

Since we use a probabilistic assignment for the cells, we may face to collisions. If a cell is allocated to two interfering transmitters, the collisions are repetitive: they occur during every slotframe if a packet has to be transmitted. These collisions would have a negative impact on both the reliability and the energy consumption.

A cell may undergo a low Packet Delivery Ratio because of external interference (e.g. Wi-Fi or Bluetooth) [40. TSCH relies on a slow channel hopping technique: a given cell uses a different physical frequency in the different slotframes. Thus, a cell will collide only during some slotframes, improving significantly the reliability [5].

We do not consider here extern interference since channel hopping has been proved to perform well in this kind of environment [5]. In particular, with narrow band noise, only some channels are impacted. Because TSCH uses channel hopping, only some slotframes are impacted: the impact of this kind of interference is 
only temporary. Besides, over-provisioning reserves some additional cells for the retransmissions, making the network reliable.

We focus here on detecting collisions inside the network.

\subsection{Detecting Colliding Cells}

Because of collisions, the Scheduling Function will reserve additional cells for retransmissions. Besides, multiple flows may also be forwarded by the same node to its parent. Thus, we have several outgoing cells in the slotframe.

Because of over-provisioning and retransmissions, a cell is not used in each slotframe to transmit a frame. If the same cell is allocated to two interfering transmitters, a collision will occur only if both transmitters have a frame to transmit at the same time. This collision ratio depends on the traffic model and the reliability, which impacts the number of retransmissions.

Muraoka et al. 41 already proposed a mechanism to detect collisions. In this tx-housekeeping strategy, a transmitter tries to estimate the probability of collision for a given cell by monitoring the Packet Delivery Ratio of each cell individually. The process considers a cell collides if it exhibits a significantly smaller PDR (by default 66\%) compared with the average PDR for all the cells. Thus, the cell has to be relocated: its channel offset and timeslot are released, and another cell is re-negotiated with the next hop. We reuse here this strategy to detect colliding cells.

We can note that external interference impacts also the reliability. However, because each cell uses a pseudo-random hopping sequence, external interference impacts equally all the channel offsets. Moreover, the traffic profile (inter packet time, transmission durations) is probably different for external traffic, and will with high probability impact equally all the different timeslots. Thus, this collision detection is triggered only by internal interference, which could be solved with a relocation strategy in the schedule. We propose consequently to adopt here the same approach.

Because the success rate is a stochastic value, we compute the WMEWMA of the Packet Delivery Ratio (PDR). This estimators helps to filter data and to smoothen the variations. More precisely, a node monitors the ratio of packets correctly acknowledged during a time window. At the end of this time interval, it computes the radio link quality metric as:

$$
\operatorname{PDR}_{W M E W M A, i}(N)=\alpha \cdot P D R_{W M E W M A, i-1}(N)+(1-\alpha) \frac{N_{t x}(N)}{N_{a c k}(N)}
$$

where $N_{t x}(N)$ is the number of packets transmitted to $N$ during the last time window, $N_{a c k}(N)$ the number of ack received from $N$ during the same interval, and $\alpha \in[0,1]$ is a constant, which controls the effect of the previously estimated value on the new one.

Finally, each node executes the following procedure (algo 3):

1. After each transmission, the transmitter updates the number of transmitted packets

2. Periodically, for each neighbor, a node tries to identify the colliding cells:

(a) it computes the average PDR for a given neighbor (lines 4-7) using the eq 18

(b) if the cell $c$ has a PDR inferior than the following limit, it is considered as colliding (line 9):

$$
\operatorname{PDR}(c) \leq \bar{x} \cdot\left(1-\Delta_{\text {coll }}\right)
$$

with $\operatorname{PDR}()$ representing the packet delivery ratio associated with a cell, $\bar{x}$ the average PDR among all the cells, and $\Delta_{\text {coll }}$ a constant.

We will present below experimental results which validate this simple approach. Thanks to channel hopping, we are able to make the network stable, and to detect collisions efficiently. 


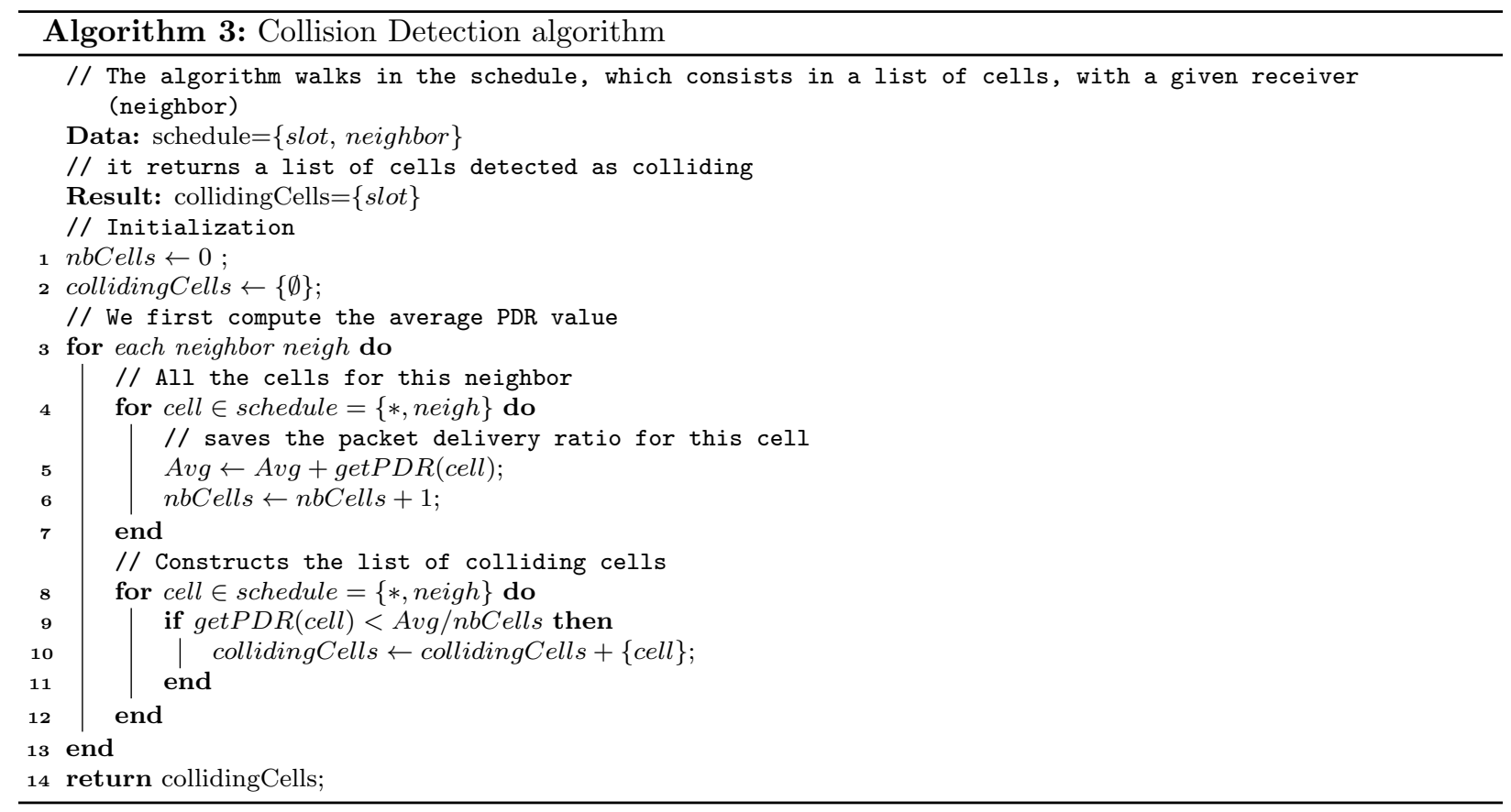

\subsection{Schedule Rearrangement}

SF0 [15] uses an hysteresis function to maintain the number of scheduled cells almost equal to the number of packets to transmit. We use here rather a variant SFloc [17, which takes into account lossy links. The method is agressive, and reserves new cells as soon as its buffer becomes larger than the number of outgoing cells (for each track), whatever the reason is. If a cell collides, many retransmissions occur and tend to let the packet stay for a longer time in the queue. Thus, SFloc allocates more cells to the same neighbor to deliver all the packets of the queue before the end of the slotframe.

SFloc also proposes to inversely deallocate the cells when it considers it has too much bandwidth (i.e. some cells are not used). We describe here how we modified SFloc to cope with colliding cells. First, a deallocation is triggered for a track either when a cell is not used for a long time, or when a subset of the cells is always sufficient to empty the queue before the end of the slotframe (this cell is virtually useless). For instance, a cell has a large ETX and becomes de facto useless to deliver reliably the packet to the neighbor. Finally, the node deallocates the cell which provides the lowest PDR for this track, it is probably one of the colliding cells if some of them collide.

This simple Scheduling Function has been proved to perform quite well in complex situations (external interference, with possible routing reconfigurations during the convergence period) [17]. However, any Scheduling Function can be here adapted, and our approach is quite orthogonal, selecting actually which cells to allocate.

\section{Experimental Performance Evaluation}

We evaluate here the performance of our stratum scheduling strategy to reduce the end-to-end delay. While several centralized algorithms have been proposed in the literature, to the best of our knowledge, none of them has been evaluated experimentally.

As discussed in section 2.3 the centralized scheduling algorithms have many implicit assumptions concerning the traffic, radio topology, radio link qualities, etc. Practically, this control information is complicated to collect in a reliable manner. Moreover, the conditions may continuously evolve. Unfortunately, no centralized algorithm describes how to patch their schedule to deal with these changes. 


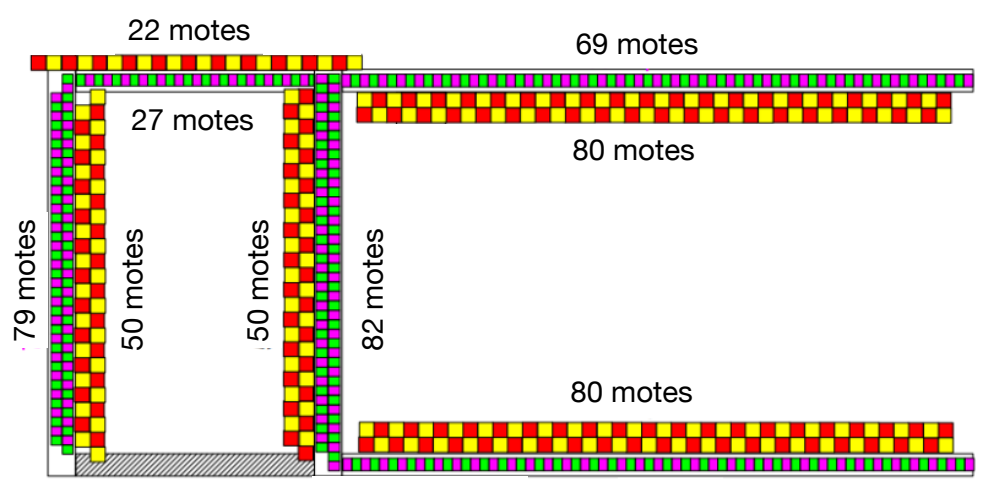

69 motes

Figure 6: Topology of the Grenoble's IoT-Lab Testbed

For all these reasons, we didn't compare our approach with a centralized algorithm, which would require strong adaptations to be executed on top of any radio topology. We compare rather the following approaches:

random: we use here the default approach of SF0, picking a random slot and channel offset in its schedule in its $6 \mathrm{P}$ requests;

stratum: we implement our stratum approach, exploiting independent blocks for each stratum. Each node extracts from the EB the number of and the size of each stratum to pick randomly a cell available in its block.

Stratum is independent on the Scheduling Function (SF) used in 6TiSCH: while the SF counts the number of cells to reserve (or deallocate), Stratum selects the channel offset/timeslots to use. Thus, our approach is very orthogonal with the SF.

\subsection{Experimental setup}

Our performance evaluation relies on the FIT-IoT lab platform (https://www.iot-lab.info/), and in particular the Grenoble's indoor testbed, where motes are placed in corridors (false ceilings and floors, cf. Fig 66. The testbed comprises motes based on a STM32 (ARM Cortex M3) micro-controller (ST2M32F103REY). Each mote embeds a AT86RF231 radio chipset, providing an IEEE802.15.4 compliant PHY layer.

The IoT-Lab testbed belongs to the real-world testbed category, since several WiFi Access Points (APs) are deployed in the building. Under such a realistic indoor environment i.e., a typical office space, the nodes are the object of external interference originated from wireless devices, such as Wi-Fi or other IEEE8021.5.4PHY compliant networks.

We integrated our stratum strategy in the openWSN (https://openwsn.atlassian.net/) implementation of the 6 TiSCH stack. It provides an open-source implementation of IEEE802.15.4e-TSCH, 6P, SF0, 6LoWPAN, RPL. Table 2 contains the experiments parameters.

\subsubsection{Traffic Isolation with 6 TiSCH}

We use here a version implementing tracks, distributed scheduling, etc. 17,1 The best-effort track uses shared slots (with contention) for the RPL control packets (e.g. DIO, DAO) and to send the packet requests to negotiate new dedicated slots $(6 \mathrm{P})$. Besides, each packet generated by an application is attached to a particular track (a track id of 16 bits, and a track owner of 64 bits): a flow reserves hop-by-hop its own dedicated resource, with dedicated slots without contention. A more detailed description may be found in 17.

\footnotetext{
${ }^{1}$ the branch "track" of https://github.com/ftheoleyre/openwsn-fw/ and https://github.com/ftheoleyre/openwsn-sw/ is freely available
} 


\begin{tabular}{|l|c|}
\hline Parameter & Value \\
\hline Experiment duration & $300 \mathrm{~s}$ \\
Traffic type, rate & CBR, 5 pkts $/$ min \\
Data packet size & 127 bytes \\
Number of nodes & 20 nodes \\
\hline MAC layer & IEEE802.15.4-TSCH \\
Type of cells & softcells (distributed) \\
Time slot duration & $15 \mathrm{~ms}$ \\
Slotframe length & 101 slots \\
\hline Routing protocol & RPL \\
Routing metric & ETX \\
\hline Radio chipset & AT86RF231 \\
Radio chipset & STM32F103REY (ARM cortex-M3) \\
\hline
\end{tabular}

Table 2: Experiment parameters

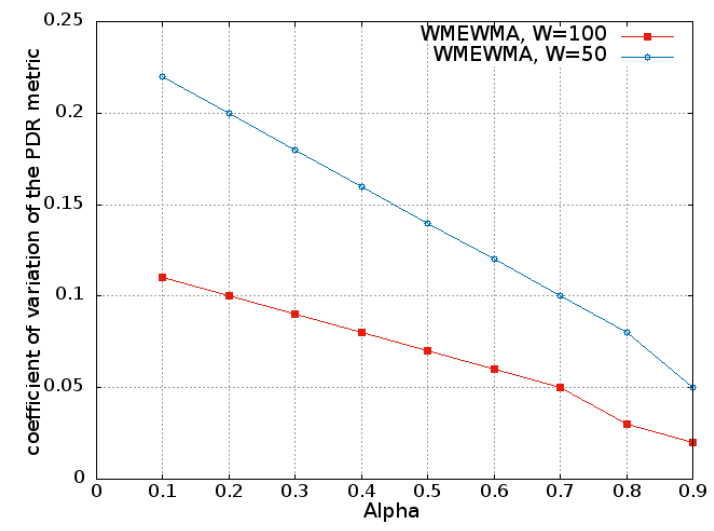

Figure 7: Impact of the memory constant $(\alpha)$ in the WMEWMA estimator

\subsection{WMEWMA performance}

We evaluate here the efficiency of the WMEWMA filter to detect cells with a bad PDR. We first measure the impact of the history window (number of samples to compute the average PDR metric). We compared a short-term $(\mathrm{w}=50)$ and long-term $(\mathrm{w}=100)$ metric: a large history window means a better stability, but also less reactivity. Similarly, we studied also the impact of the $\alpha$ (alpha) factor in equation 18 .

Figure 7 illustrates this impact. With a shorter window $(\mathrm{w}=50)$, we have a larger volatility: the PDR value changes significantly, the packet losses representing a stochastic variable. In the same way, a smaller $\alpha$ means we give an higher priority to the last measured value. Thus, using either a larger window $(\mathrm{w}=100)$ or a large alpha $(\alpha=0.9)$ value is required to smoothen this PDR metric, and to not over-react to local and temporary changes.

Then, we compared the performance with and without WMEWMA in Figure 8 . We measures the Packet Delivery Ratio for the packets transmitted to the sink. The WMEWMA estimator helps to avoid useless oscillations which lead to schedule modifications. Thus, the network converges more quickly, increasing finally the average Packet Delivery Ratio for the data packets received by the sink.

\subsection{Efficient Collision Detection}

We evaluated the efficiency of the collision detection mechanism in a very simple scenario. Indeed, this mechanism was proposed in Muraoka et al. [41, but to the best of our knowledge, no paper presents an 


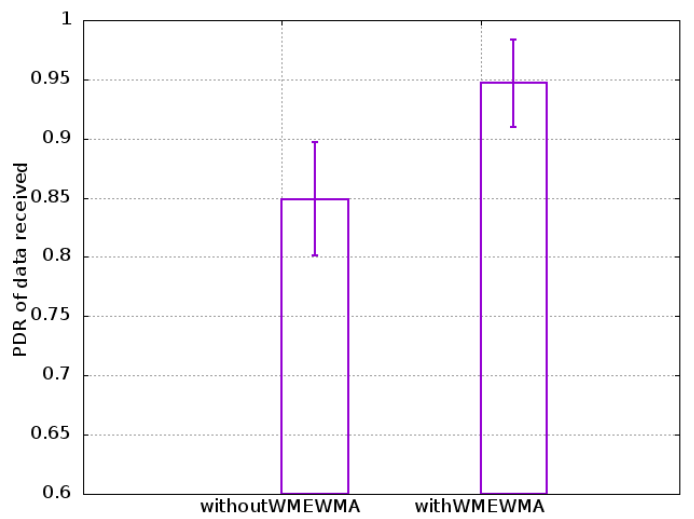

Figure 8: Performance comparison in terms of Packet Delivery Rate(PDR), with and without WMEWMA (20 nodes, w=50, $\alpha=0.9)$

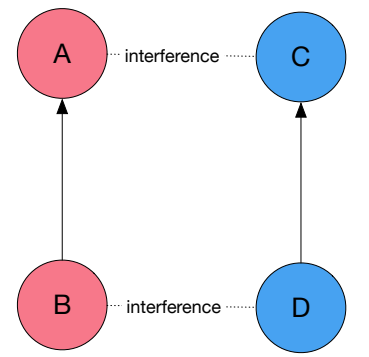

Figure 9: Topology to evaluate the collision detection mechanism

experimental validation. Two pairs of nodes are selected in the testbed (Figure 9): they are mutually interfering, and we fix manually their schedule (hard-coded schedule). Each pair of nodes has 10 cells, and we vary the number of colliding cells (between 0 and 10). We implement a CBR flow (5 packets/minute): some cells may be unused, depending on the collisions and the retransmissions.

The IoT-Lab testbed is a shared facility: Wi-Fi Access Points and other IEEE802.15.4 networks are colocated, generating external interference. Thus, we also stress the collision detection mechanism, to prove it works properly, even in presence of other colocated and uncontrolled networks.

\subsubsection{Collision detection accuracy}

In order to assess the accuracy of this detection mechanism, we quantify the number of wrong decisions:

False Positive: a cell is detected as colliding although the other pair does not use this cell;

False Negative: a cell isn't detected as colliding although the other pair uses also this cell;

We can see that when the number of colliding cells is small (less than 3 cells), a node detects very efficiently the collisions (Figure 10a). Indeed, the colliding cells present a very different packet delivery ratio (more retransmissions are required), and the difference is considered significant. The number of false positive and negative begins to increase with more than one third of colliding cells. However, some cells are even detected as colliding (true positive), and they may be re-allocated: step by step, the number of colliding cells will decrease. 


\begin{tabular}{|c|c|c|c|}
\hline \multirow{2}{*}{ Nb. of nodes } & \multirow{2}{*}{$\begin{array}{c}\text { Ratio of cells allocated } \\
\text { several times }\end{array}$} & \multicolumn{2}{|r|}{ Colliding } \\
\hline & & Stratum Id & Dist. of transmitters \\
\hline 30 & 0.029 & 6 & $4 \mathrm{~m}$ \\
\hline 40 & 0.022 & 4 & $3 \mathrm{~m}$ \\
\hline 50 & 0.037 & $5 \& 6$ & $4 \mathrm{~m}$ \\
\hline
\end{tabular}

Table 3: Ratio of cells allocated to different transmitters (and which may create possibly a collision) - quantification of the severity of collisions

Then, we measured the impact of the link quality on the accuracy (Figure $10 \mathrm{~b}$ ). We consider here 5 colliding cells (some false positive and negative begin to occur). Obviously, having less reliable radio links impacts the detection mechanism. However, we consider that the accuracy remains largely acceptable.

Always with the aim of measuring the impact of the link quality on the accuracy, we measured the PDR by varying the distance between the source and the destination for 5 colliding cells (Figure 11). As illustrated, we confirme the previous results by achieving an acceptable PDR values.

\subsubsection{Convergence}

Then, we studied the convergence of the scheduling process: cells are re-allocated to deal with collisions, but the schedule has to become stable after a while. In figure 12, we report the number of cells detected as colliding during a time window of 30 minutes, measured during 2 hours. We represent the boxplots for all the experiments. At the beginning, many cells are colliding, and SFloc will ask to re-allocate them. However, after 2 hours, we can verify that SFloc converged to a legal state: no cell collides anymore.

\subsubsection{Collision Detection in Large Networks}

We also evaluate the efficiency of the collision detection mechanism in a a larger network, comprising up to 50 nodes. For each experiment, we collect the schedule installed in each mote, and compute the ratio of cells which collide, i.e. at least two radio links use the same pair of channel offset / timeslot (Tab. 3). We can see that the ratio of colliding cells is very low: at most $4 \%$ of cells are allocated at least twice in the network. Increasing the number of nodes also increases this probability: we have more cells allocated since the number of packets to forward is also larger.

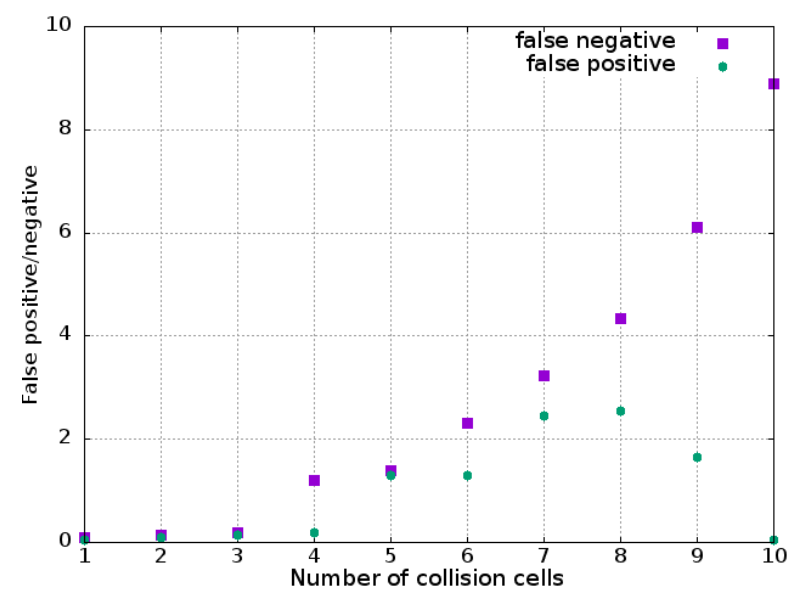

(a) Nb. of colliding cells (dist. src/dest=5 hops)

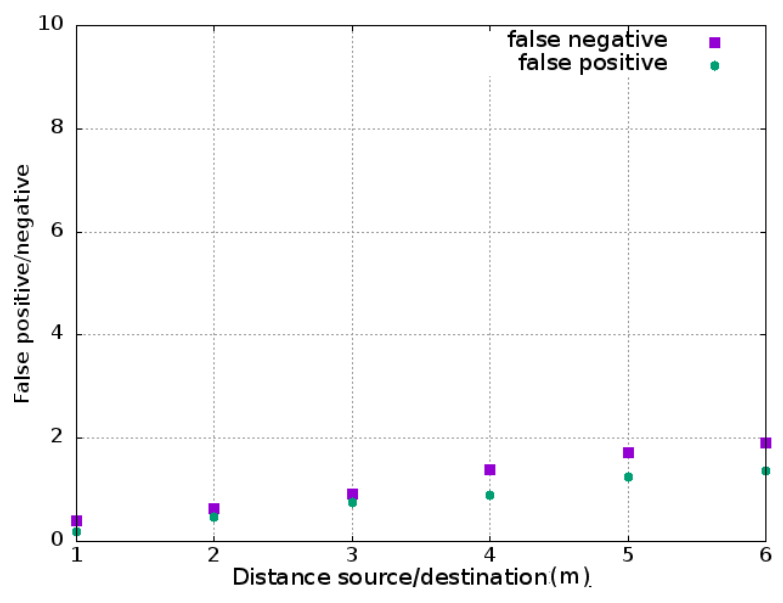

(b) Source sink distance (5 colliding cells)

Figure 10: False Positives/Negatives with 1 source, $\mathrm{CBR}=5$ pkts/min, 20 cells in the schedule, 


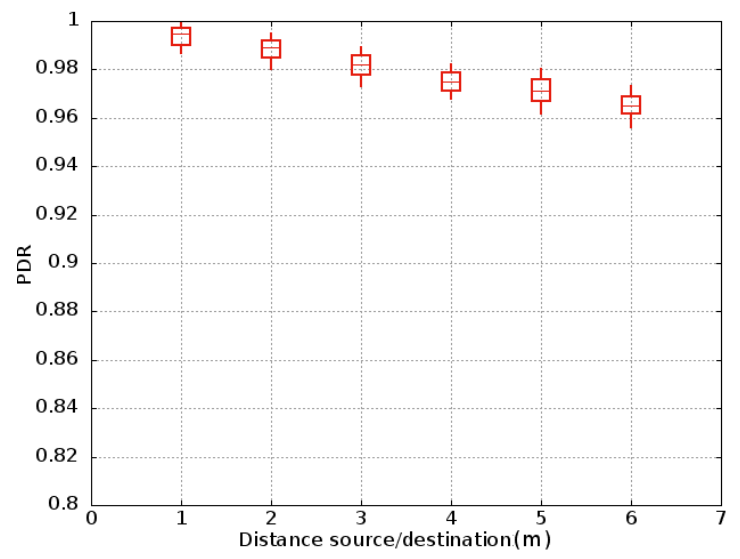

Figure 11: Packet delivery ration Vs. distance source/destination

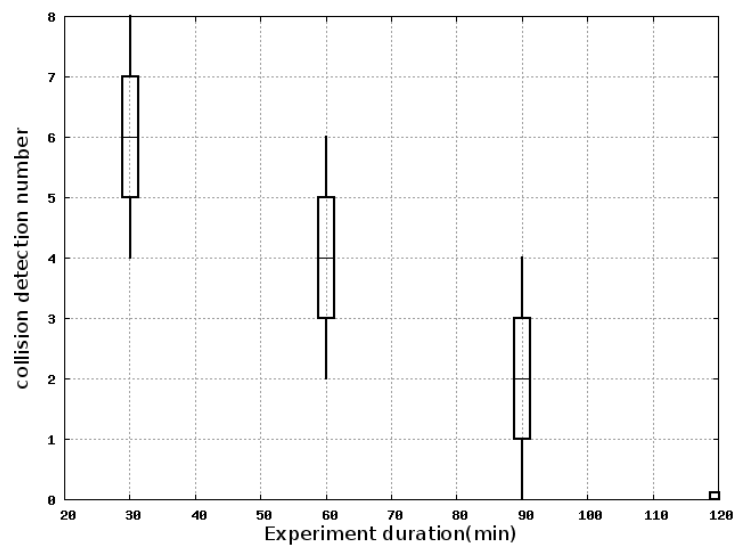

Figure 12: Number of colliding cells detected during a time window of 30 minutes (five nodes, hard schedule with 10 collision cells at $\mathrm{t}=0, \mathrm{CBR}$ value $=5 \mathrm{pkts} / \mathrm{min}$ )

Then, we tried to identify the severity of these collisions. Indeed, using the same cell may not create collisions if the two radio links are sufficiently far from each other. The reactive approach to detect collisions obviously works only if the corresponding transmitters collide actually. Let consider the figure 4 the nodes in the first stratum will for sure collide if they use the same cell. This is obviously not obligatory the case in the largest stratums. Tab. 3 references also the stratum id concerned by the colliding cells: in all the cases, this corresponds to the largest stratums. Besides, the transmitters are quite far from each other (3 or 4 meters). After analyzing the logs (packet received/transmitted for each node), we concluded that the cells were allocated twice, but didn't correspond to collisions: the capture effect is sufficient to avoid packet losses.

\subsection{Multihop Network}

Experimental results are considered for the default IEEE802.15.4-TSCH operating with a superframe length of 101 slots, a duration of a single slot $=15 \mathrm{~ms}$, number of nodes $=20, \mathrm{CBR}$ value $=5 \mathrm{pkts} / \mathrm{min}$.

\subsubsection{Topology of 20 nodes}

Figure 13a illustrates the end-to-end reliability when considering a multihop network of 20 nodes. We can note that both scheduling strategies achieve the same reliability: only $5 \%$ of the flows have a reliability lower than 85\%: they concern sources which suffer from local interference, with a negative impact on the 
PDR. Indeed, the IoT-Lab testbed is a shared facility: concurrent experiments run, and generate external interference. However, three quarters of the flows still have an end-to-end reliability larger than $95 \%$.

We measured the packet delivery ratio achieved by the different flows depending on their distance to the border router (Figure 13b). We group the source nodes according to their geographic distance to the border router. Neighbors of the border router achieve a reliability equal to $99 \%$ and $98 \%$ for the random and stratum strategies respectively. A node far apart from the border router will probably use a multihop route, with a lower reliability. We can highlight that surprisingly, TSCH ensures a very good end-to-end reliability, even for the farthest nodes. The approach is consequently highly scalable: we can guarantee a packet delivery ratio of even $95 \%$ for the nodes 35 meters far from the border router (a route with 6 or 7 hops). This demonstrates the scalability of the $6 \mathrm{TiSCH}$ stack for industrial wireless networks.

The scheduling algorithms are able to provide a low network duty cycle, DC, computed as the percentage of timeslots during which a node is active (transmitting or receiving data). Fig. 13c presents the Duty Cycle Ratio according to the source border router geographic distance. We maintain a reasonable low duty cycle ratio. Obviously, the ratio of active timeslots increases when the distance between source and destination exceeds 35 meters (7 hops).

We also measured the end-to-end delay (Figure $13 \mathrm{~d}$ ). We represented the cumulative distribution function

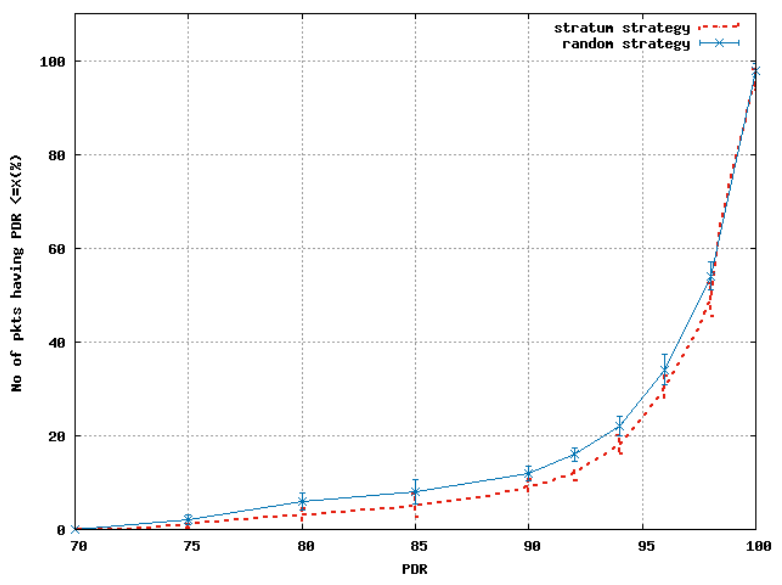

(a) Cumulative Distribution Function of the PDR among the flows

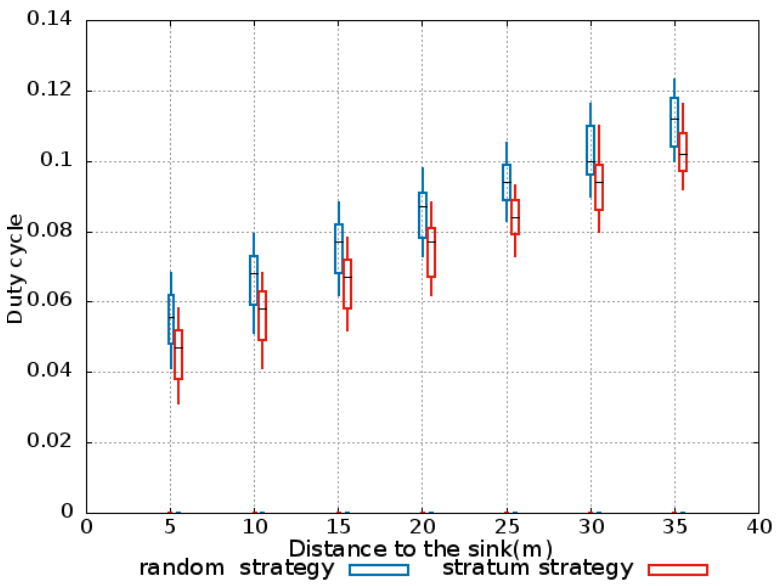

(c) Average Duty Cycle Ratio

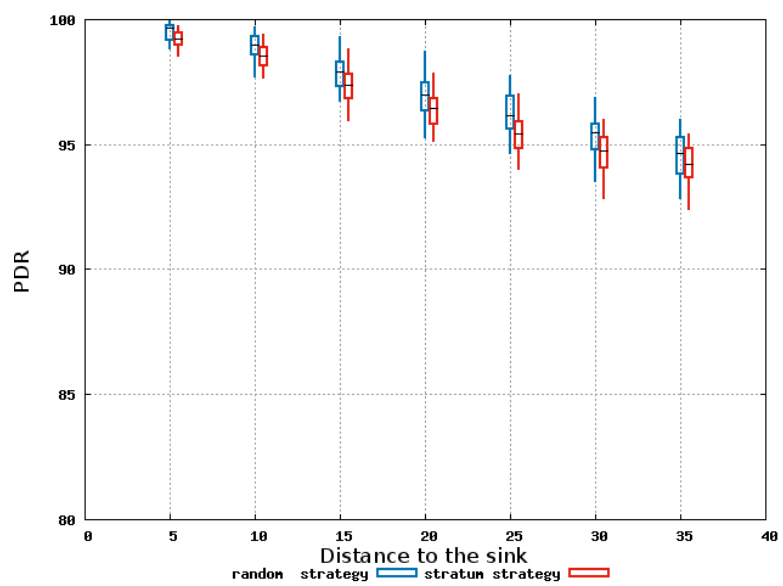

(b) Impact of the end-to-end distance on the reliability

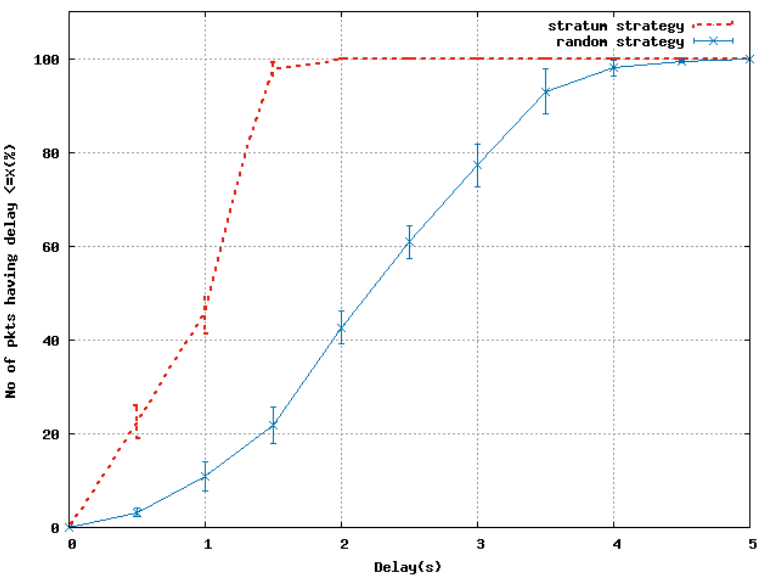

(d) CDF of the end-to-end delay

Figure 13: Network with 20 nodes, diameter of 8 hops, CBR of 5 pkts/min. 


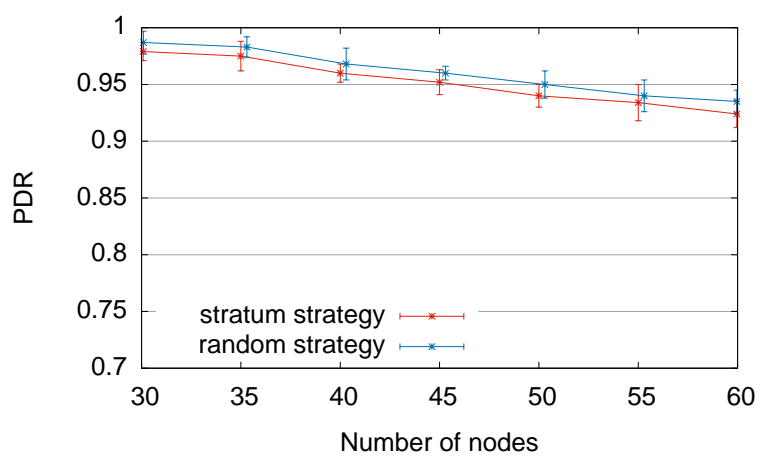

(a) PDR vs. nb. of nodes $(\mathrm{CBR}$ value $=5 \mathrm{pkts} / \mathrm{min})$

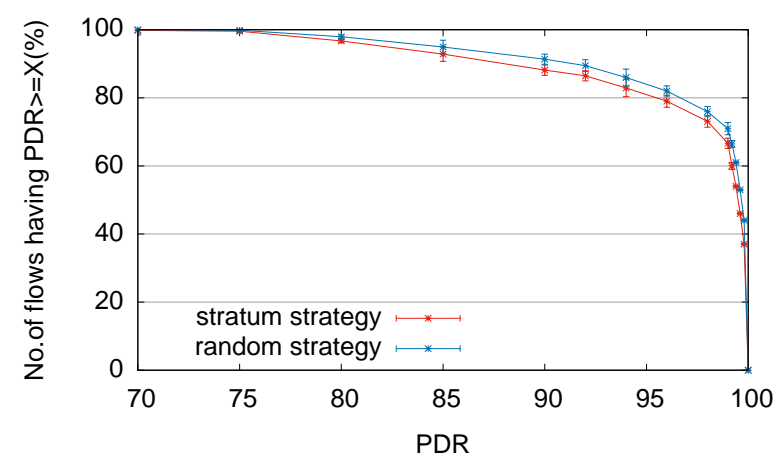

(b) Complementary CDF of the end-to-end PDR (60 nodes

Figure 14: CBR of 5 pkts/min, diameter of 10 hops

to also consider the worst case. The stratum strategy is very efficient: while it achieves a similar reliability as the random strategy, it reduces significantly the end-to-end delay. The stratum strategy achieves a delay of 1.5 seconds in the worst case, while the random strategy presents a worst case delay of 4.5 seconds $(+300 \%)$.

Even for long routes (6 or 7 hops in this instance), the network achieves to deliver all the packets within the slotframe (equal here to 1.5 seconds). We are consequently able to provide a distributed scheduling solution with a high reliability while upper-bounding the end-to-end delay.

In conclusion, we succeed to reduce the end-to-end delay while not impacting the reliability, and presenting also the same duty cycle ratio as a random strategy.

\subsubsection{Topology of 30 to 60 nodes}

We consider now larger cluster tree topologies, comprising between 30 and 60 nodes. We select 10 groups of 3 (respectively 6) nodes along a corridor (cf. Fig. 6), leading to a line topology. Each group is separated by 3 meters, so that the network diameter is limited to 10 hops. On average, each node has 3 (respectively 6) neighbors, the nodes of the same group being located as close as possible to each other.

Figure 14a illustrates the scalability of the two scheduling algorithms. We can note that both scheduling strategies still achieve an end-to-end PDR larger than 93\%, even with 60 nodes. Besides, both strategies behave very similarly: we succeed to reduce the end-to-end delay with a limited impact on the reliability.

Then, we also measured the distribution of the PDR for all the flows with a topology of 60 nodes (Fig. 14b). As we can note, $80 \%$ of the flows have an end-to-end reliability larger than $95 \%$. Some flows exhibit a lower PDR, mainly due to the external interference (i.e., other experiments are executed simultaneously on the same testbed). The corresponding links exhibit a larger PER, with a negative impact on the e2e reliability. In conclusion, the stratum strategy seems scalable.

\subsection{Correlation End-To-End Reliability / Geographical Distance}

We can remark a strong correlation between the distance and the end-to-end PDR in the previous graphs. We propose here a more precise evaluation of this correlation. To verify the independency of the PDR and the distance to the border router, we use the $K h i^{2}$ test. The test leads to $\chi^{2}=33.38$. The $K h i^{2}$ table leads us to conclude that there is a dependency between the two parameters with $0.5 \%$ chance of being wrong.

The correlation coefficient is calculated as:

$$
r=\frac{\operatorname{cov}(x, y)}{\sqrt{\operatorname{var}(x) \operatorname{var}(y)}}
$$

where, $x$ presents the distance and $y$ the PDR.

Since $\bar{x}=20, \bar{y}=96.42, n=7$ :

$$
r=-0.94
$$


We conclude that it is a strong negative linear correlation, so the equation is of the form $y=a x+b(\mathrm{cf}$. Appendix A for the numerical details).

$$
\begin{gathered}
a=\frac{\operatorname{cov}(x, y)}{S_{x}^{2}} \\
S_{x}^{2}=\frac{1}{n} \sum_{i=1}^{n}\left(x_{i}-\bar{x}\right)^{2} \\
b=\bar{y}-a \bar{x} \\
y=-0.18 x+92
\end{gathered}
$$

In conclusion, the correlation coefficient is quite low $(a=-0.18)$. This demonstrates this scheduling solution scales quite well: even when the source is several hop far from the border router, its packet delivery ratio is reduced but remains acceptable (the PDR decreases by 1,9\% per meter).

\subsection{Heavy Traffic Scenario}

We then studied the impact of the traffic load. We measured the packet delivery ratio with an interpacket time comprised between $700 \mathrm{~ms}$ and $1.4 \mathrm{~s}$ (Fig. 15a). With an high load (700ms), more packets are lost: the network does not have enough cells to forward all the packets.

For 20 nodes which transmit packets with an inter packet time $=700 \mathrm{~ms}$ using 20 cells and a slot time $=15 \mathrm{~ms}$, the load without multihop is: $20 *(20 * 15) / 700$, which means that the number of cells required without retransmission is equal to 9 cells.

However, both the random and the stratum strategies achieve the same reliability: the size of the different stratums is accurately chosen, and our re-arrangement solution is efficient to deal with collisions.

When the inter-packet time exceeds 1.2 seconds (i.e. around one packet per slotframe), the network is able to guarantee ultrahigh reliability, and the PDR is almost equal to $100 \%$ for all the flows.

We measured similarly the end-to-end delay (Figure 15b) with an inter-packet time of 700ms (high traffic condition). The stratum strategy keeps on guaranteeing a better average and worst delay. In the worst case, the stratum strategy delivers the packets in 5 seconds (3 slotframes): too many retransmissions are required, and they don't fit in a single slotframe. While the end-to-end delay guarantee (i.e. delivery before the end of the slotframe) cannot be fulfilled when the traffic exceeds the network capacity, it keeps on outperforming the random strategy.

Finally, we can conclude the stratum strategy is robust and presents an high reliability while delivering the packets more quickly than the random strategy. Because schedules are computed distributively, the traffic may exceed the network capacity: a call admission (either static or dynamic) is consequently recommended.

Finally, we measure the average duty cycle ratio (the ratio of timeslots during which a node is active) with different traffic conditions (Fig. 15c). When each node generates several packets per second (on the right), the duty cycle ratio is much higher: around $40 \%$ when each node generates 6 packets per second. These stressful conditions have obviously a strong impact on the energy consumption. With a low traffic (a few packets per minute), a node maintains a duty cycle ratio below $1 \%$ (even for the most active node). In this more common case, we are able to guarantee a large network lifetime.

\subsection{Large-scale topology with 100 nodes}

We now consider a large-scale cluster tree topology with 100 nodes, and observe the Complementary Cumulative Distribution Function (CCDF) of the of the end-to-end PDR (Fig 16a). A large topology has an impact on the reliability: more packets have to be transmitted, and create possibly more collisions. However, the random and stratum strategies exhibit similar results: the collisions seem solved efficiently by the rescheduling policy. However, a long route means also more retransmissions, and possibly a larger probability to drop the packet before it is received by the destination. 


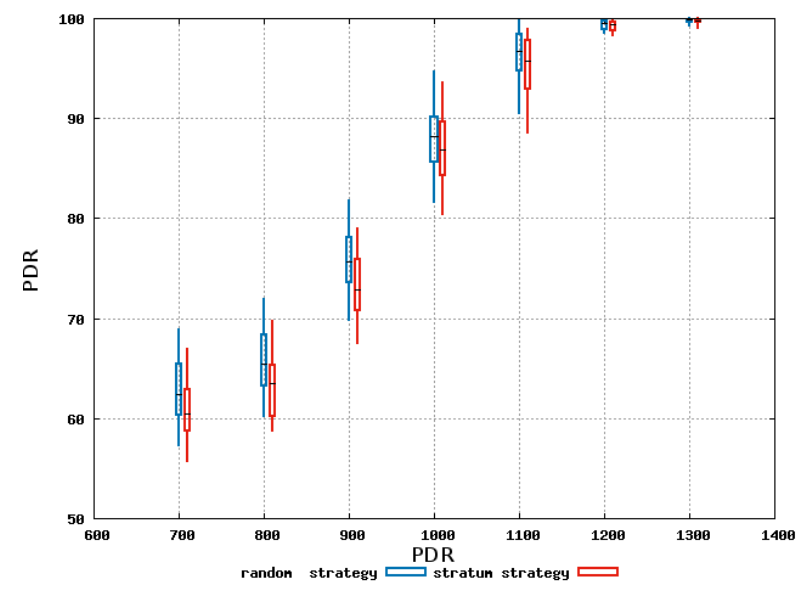

(a) Impact of the traffic load on the end-to-end Packet Delivery Ratio

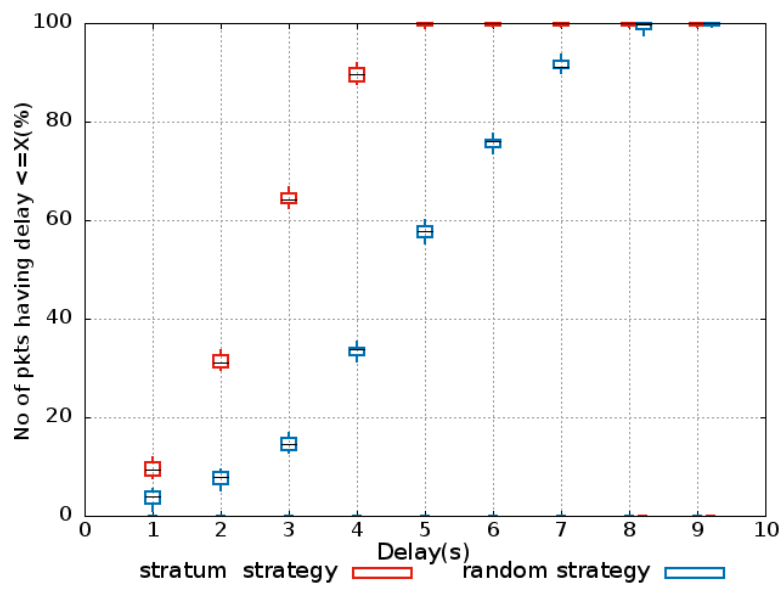

(b) CDF of the end-to-end delay (inter-packet time $=700 \mathrm{~ms}$ )

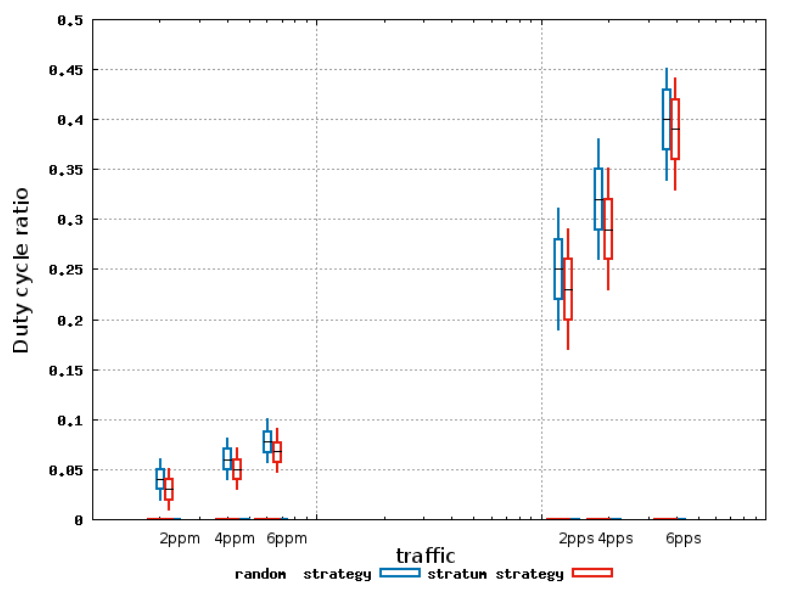

(c) Average Duty Cycle Ratio for different traffic conditions (a few packets per second versus a few packets per minute)

Figure 15: Heavy vs. Light traffic conditions

We then change the traffic intensity to evaluate the ability of the network to still provide long routes when the nodes have less packets to forward (Fig. 16b). When the traffic load is very high, more collisions occur: not enough cells are available in the scheduling matrix to forward all the packets, we reach the network capacity. However, for light traffic conditions, more packets can be delivered. We can conclude that a distributed scheduling solution is relevant only for medium-scale networks or light traffic conditions. Else, too many cells have to be provisioned, and the probability to create collisions is too high, impacting negatively the convergence and the reliability.

\subsection{Concluding remarks}

The stratum scheduling approach is very efficient to set-up easily a TSCH network, without relying on a central controller to compute and to distribute the schedule. While the initial block sizes are computed according to geometric considerations, this size may be adapted later, when too many collisions are for instance detected in a stratum. Different block sizes may even be computed if we have a perfect a priori knowledge of the deployment (topology and traffic). It is robust to collisions and changes, the Scheduling Function monitors continuously the performance, detecting collision, and re-allocating the cells in the correct blocks. To our mind, such distributed strategy is particularly recommended for medium-scale networks, with reasonable traffic. 


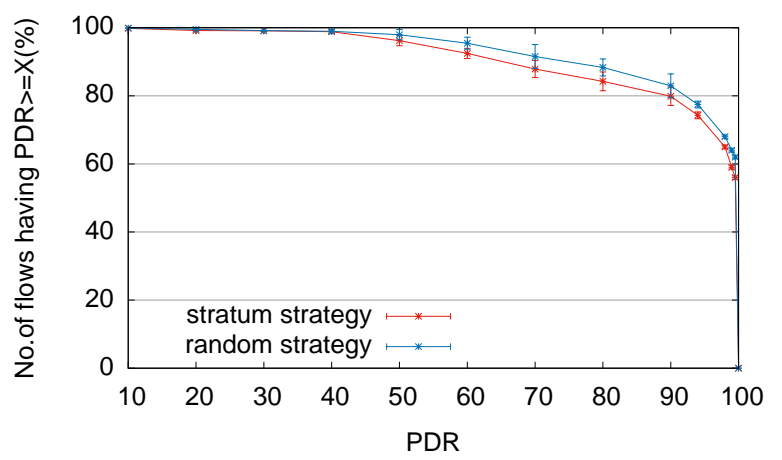

(a) Complementary CDF of the end-to-end PDR (with 100 nod inter packet time=12s)

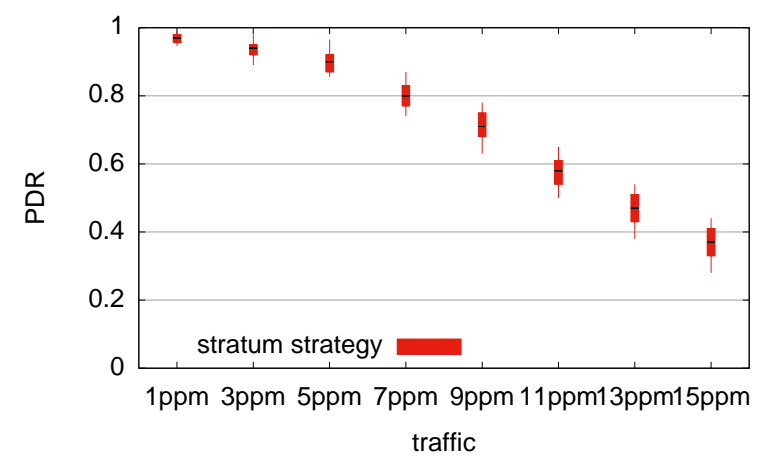

(b) Impact of the traffic load on the end-to-end reliability (with 100 nodes and 1 sink)

Figure 16: Large-scale topology with 100 nodes and 1 sink, slotframe with 203 cells

For very large-scale networks with a very high traffic to forward, the network must operate close to its duty cycle limit. Thus, more collisions occur, which slow down the convergence of the relocation algorithm. A centralized schedule would help to improve the performance, but it requires to know precisely the radio topology, the interfering links, the link qualities, which still constitute a challenging problem in many situations (cf. section 3.1).

\section{Conclusion and Future Work}

We presented here a distributed scheduling solution designed for $6 \mathrm{TiSCH}$. It allocates the soft cells autonomously and minimizes the end-to-end delay: a packet present in the buffer at the beginning of the slotframe should be delivered before the end. We use a stratum scheduling strategy, dividing the whole schedule into blocks. Then, all the nodes equidistant from the border router share the same block to minimize the buffering delay. By selecting appropriately the size of each block, we are able to guarantee a packet is delivered with a slotframe to the border router, even if it is multihop far away. We re-used the self-healing mechanism of SF0 to detect colliding cells, and evaluated its efficiency experimentally: an WMEWMA estimator helps to smoothen the variations, reducing the number of false positives / negatives.

In a future work, we plan to propose a call admission mechanism. Indeed, the network begins to perform poorly when the network capacity is exceeded. We must guarantee on the contrary that the existing flows are not perturbed by the new ones (i.e. traffic isolation). We also plan to compare stratum, SF0 and SFloc with a centralized algorithm. However, we have to propose the mechanisms to collect all the information required for the scheduler (radio links, their quality, traffic volume, etc.) Moreover, we also have to provide the protocols to update the schedule on-the-fly, when e.g. a new node is inserted in the network and has to send packets.

\section{Acknowledgement}

This work was partly supported by the University of Strasbourg Idex project Diag@IoT.

\section{References}

\section{References}

[1] I. Hosni, F. Théoleyre, N. Hamdi, Localized scheduling for end-to-end delay constrained Low Power Lossy networks with 6TiSCH, in: International Symposium on Computers and Communication (ISCC), IEEE, 2016. doi:10.1109/ISCC.2016. 7543789. 
[2] L. Atzori, A. Iera, G. Morabito, The internet of things: A survey, Computer Networks 54 (15) (2010) $2787-2805$. doi:10.1016/j.comnet.2010.05.010

[3] L. D. Xu, W. He, S. Li, Internet of things in industries: A survey, IEEE Transactions on Industrial Informatics 10 (4) (2014) 2233-2243. doi:10.1109/TII.2014.2300753

[4] IEEE Std 802.15.4e-2012, IEEE Standard for Local and metropolitan area networks-Part 15.4: Low-Rate Wireless Personal Area Networks (LR-WPANs) Amendment 1: MAC sublayer (2012).

[5] T. Watteyne, A. Mehta, K. Pister, Reliability through frequency diversity: Why channel hopping makes sense, in: ACM Symposium on Performance Evaluation of Wireless Ad Hoc, Sensor, and Ubiquitous Networks (PE-WASUN), 2009, pp. 116-123. doi:10.1145/1641876.1641898.

[6] M. Palattella, N. Accettura, X. Vilajosana, T. Watteyne, L. Grieco, G. Boggia, M. Dohler, Standardized protocol stack for the internet of (important) things, IEEE Communications Surveys Tutorials 15 (3) (2013) 1389-1406. doi:10.1109/ SURV.2012.111412.00158

[7] D. Dujovne, T. Watteyne, X. Vilajosana, P. Thubert, 6tisch: deterministic ip-enabled industrial internet (of things), IEEE Communications Magazine 52 (12) (2014) 36-41. doi:10.1109/MCOM.2014.6979984.

[8] T. Winter, P. Thubert, A. Brandt, J. Hui, R. Kelsey, P. Levis, K. Pister, R. Struik, J. P. Vasseur, R. Alexander, Rpl: Ipv6 routing protocol for low-power and lossy networks, rfc 6550, IETF (2012). doi:10.17487/RFC6550

[9] Q. Wang, X. Vilajosana, 6top protocol (6p), draft 4, IETF, https://tools.ietf.org/html/ draft-ietf-6tisch-6top-protocol-04 (March 2017).

[10] K. Pister, L. Doherty, Tsmp: Time synchronized mesh protocol, in: Parallel and Distributed Computing and Systems, 2008.

[11] J. N. Tsitsiklis, K. Xu, On the power of (even a little) centralization in distributed processing, in: ACM SIGMETRICS, 2011, pp. 161-172. doi:10.1145/1993744.1993759

[12] M.R. Palattella, et al., On optimal scheduling in duty-cycled industrial iot applications using IEEE802.15.4e TSCH, Sensors Journal, IEEE 13 (10) (2013) 3655-3666. doi:10.1109/JSEN.2013.2266417

[13] A. Tinka, T. Watteyne, K. Pister, A decentralized scheduling algorithm for time synchronized channel hopping, in: ICST Transactions on Mobile Communications and Applications, 2010.

[14] N. Accettura, M. Palattella, G. Boggia, L. Grieco, M. Dohler, Decentralized traffic aware scheduling for multi-hop low power lossy networks in the internet of things, in: World of Wireless, Mobile and Multimedia Networks (WoWMoM), 2013 IEEE 14th International Symposium and Workshops on a, 2013, pp. 1-6. doi:10.1109/WoWMoM.2013.6583485

[15] D. Dujovne, L. A. Grieco, M. R. Palattella, N. Accettura, 6tisch 6top scheduling function zero (sf0), draft 4, IETF, https://tools.ietf.org/html/draft-ietf-6tisch-6top-sf0-04 (July 2017).

[16] C.-Y. Wan, S. B. Eisenman, A. T. Campbell, J. Crowcroft, Siphon: Overload traffic management using multi-radio virtual sinks in sensor networks, in: Conference on Embedded Networked Sensor Systems (SenSys), ACM, 2005, pp. 116-129. doi:10.1145/1098918.1098931

[17] F. Theoleyre, G. Z. Papadopoulos, Experimental validation of a distributed self-configured 6tisch with traffic isolation in low power lossy networks, in: International Conference on Modeling, Analysis and Simulation of Wireless and Mobile Systems (MSWiM), ACM, 2016, pp. 102-110. doi:10.1145/2988287.2989133.

[18] V. C. Gungor, G. P. Hancke, Industrial Wireless Sensor Networks: Challenges, Design Principles, and Technical Approaches, IEEE Transactions on Industrial Electronics 56 (10) (2009) 4258-4265. doi:10.1109/TIE.2009.2015754

[19] J. Lee, W. Kim, S.-J. Lee, D. Jo, J. Ryu, T. Kwon, Y. Choi, An experimental study on the capture effect in 802.11a networks, in: International Workshop on Wireless Network Testbeds, Experimental Evaluation and Characterization (WinTECH), ACM, 2007, pp. 19-26. doi:10.1145/1287767.1287772

[20] M. Sha, G. Xing, G. Zhou, S. Liu, X. Wang, C-MAC: Model-driven concurrent medium access control for wireless sensor networks, in: INFOCOM, IEEE, 2009, pp. 1845-1853. doi:10.1109/INFCOM.2009.5062105

[21] X. Vilajosana, K. Pister, Minimal 6TiSCH Configuration, draft 21, IETF (February 2017).

[22] Z. Chen, C. Wang, Use cases and requirements for using track in 6tisch networks, draft 0, IETF, https://tools.ietf. org/html/draft-wang-6tisch-track-use-cases-00 (September 2015).

[23] A. Ghosh, O. Incel, V. Kumar, B. Krishnamachari, Multi-channel scheduling algorithms for fast aggregated convergecast in sensor networks, in: International Conference on Mobile Adhoc and Sensor Systems (MASS), IEEE, Macau, Macau, 2009, pp. 363-372. doi:10.1109/MOBHOC.2009.5336979

[24] M. Yan, K.-Y. Lam, S. Han, E. Chan, Q. Chen, P. Fan, D. Chen, M. Nixon, Hypergraph-based data link layer scheduling for reliable packet delivery in wireless sensing and control networks with end-to-end delay constraints, Information Sciences 278 (2014) $34-55$. doi:10.1016/j.ins.2014.02.006

[25] M. Yigit, O. D. Incel, V. C. Gungor, On the interdependency between multi-channel scheduling and tree-based routing for WSNs in smart grid environments, Computer Networks 65 (0) (2014) 1 - 20. doi:10.1016/j.comnet.2014.02.025

[26] F. Dobslaw, T. Zhang, M. Gidlund, End-to-end reliability-aware scheduling for wireless sensor networks, IEEE Transactions on Industrial Informatics 12 (2) (2016) 758-767. doi:10.1109/TII.2014.2382335

[27] R. Soua, P. Minet, E. Livolant, MODESA: An optimized multichannel slot assignment for raw data convergecast in wireless sensor networks, in: International Performance Computing and Communications Conference (IPCCC), IEEE, 2012, pp. 91-100. doi:10.1109/PCCC. 2012.6407742

[28] K.-H. Phung, B. Lemmens, M. Goossens, A. Nowe, L. Tran, K. Steenhaut, Schedule-based multi-channel communication in wireless sensor networks: A complete design and performance evaluation, Ad Hoc Networks 26 (2015) 88 - 102. doi:10.1016/j.adhoc.2014.11.008

[29] J. Lee, W. C. Jeong, B. C. Choi, A multi-channel timeslot scheduling algorithm for link recovery in wireless multi-hop sensor networks, in: International Conference on Information and Communication Technology Convergence (ICTC), 2016, 


\begin{tabular}{|l|l|l|l|}
\cline { 2 - 4 } \multicolumn{1}{c|}{} & Distance & PDR & Total \\
\cline { 2 - 4 } & 5 & 99 & 104 \\
10 & 98 & 108 \\
15 & 97 & 113 \\
20 & 96 & 116 \\
& 25 & 96 & 121 \\
& 30 & 95 & 125 \\
& 35 & 94 & 129 \\
\hline Sum & 140 & 675 & 815 \\
\hline
\end{tabular}

(a) $\mathrm{T}$

\begin{tabular}{|c|c|c|}
\hline & Distance & PDR \\
\hline & 165.37 & 172.39 \\
\hline & 73.10 & 89.11 \\
\hline & 19.44 & 20.97 \\
\hline & 0.006 & 0.004 \\
\hline & 33.40 & 17.72 \\
\hline & 89.68 & 72.59 \\
\hline Sum & 172.92 & 164.86 \\
\hline
\end{tabular}

(c) $R^{2}$

\begin{tabular}{|l|l|}
\hline Distance & PDR \\
\hline 17.86 & 86.13 \\
18.55 & 89.44 \\
19.41 & 93.58 \\
19.92 & 96.07 \\
20.78 & 100.21 \\
21.47 & 103.52 \\
22.15 & 106.84 \\
\hline
\end{tabular}

(b) $T_{0}$

\begin{tabular}{|l|l|l|l|}
\cline { 2 - 4 } \multicolumn{1}{c|}{} & Distance & PDR & Total \\
\hline 9.25 & 2 & 11.25 \\
3.94 & 0.99 & 4.93 \\
1.00 & 0.22 & 1.22 \\
0.00 & 0.00 & 000 \\
1.60 & 0.17 & 1.77 \\
4.17 & 0.70 & 4.57 \\
& 7.80 & 1.54 & 9.34 \\
\hline Sum & 27.76 & 5.62 & 33.38 \\
\hline
\end{tabular}

(d) $R^{2} / T_{0}$

Table A.4: Table for the distance/reliability correlation

pp. 871-876. doi:10.1109/ICTC.2016.7763319

[30] T. Huynh, F. Theoleyre, W.-J. Hwang, On the interest of opportunistic anycast scheduling for wireless low power lossy networks, Computer Communications 104 (2017) 55 - 66. doi:10.1016/j.comcom.2016.06.001

[31] R. Soua, P. Minet, E. Livolant, Wave: a distributed scheduling algorithm for convergecast in IEEE 802.15.4e TSCH networks, Transactions on Emerging Telecommunications Technologies 27 (4) (2016) 557-575. doi:10.1002/ett.2991

[32] S. Duquennoy, B. Al Nahas, O. Landsiedel, T. Watteyne, Orchestra: Robust mesh networks through autonomously scheduled tsch, in: Conference on Embedded Networked Sensor Systems (Sensys), ACM, 2015, pp. 337-350. doi:10. $1145 / 2809695.2809714$

[33] M. Domingo-Prieto, T. Chang, X. Vilajosana, T. Watteyne, Distributed pid-based scheduling for 6tisch networks, IEEE Communications Letters 20 (5) (2016) 1006-1009. doi:10.1109/LCOMM.2016.2546880

[34] R. Soua, P. Minet, E. Livolant, Disca: A distributed scheduling for convergecast in multichannel wireless sensor networks, in: International Symposium on Integrated Network Management (IM), IFIP/IEEE, 2015, pp. 156-164. doi:10.1109/ INM. 2015.7140288

[35] T. P. Duy, T. Dinh, Y. Kim, Distributed cell selection for scheduling function in 6tisch networks, Computer Standards \& Interfaces 53 (2017) $80-88$. doi:10.1016/j.csi.2017.03.008

[36] T. Chang, T. Watteyne, Q. Wang, X. Vilajosana, Llsf: Low latency scheduling function for 6tisch networks, in: International Conference on Distributed Computing in Sensor Systems (DCOSS), 2016, pp. 93-95. doi:10.1109/DCOSS.2016.10

[37] D. S. J. De Couto, D. Aguayo, J. Bicket, R. Morris, A high-throughput path metric for multi-hop wireless routing, Wireless Networks 11 (4) (2005) 419-434. doi:10.1007/s11276-005-1766-z

[38] M. Haenggi, R. K. Ganti, Interference in large wireless networks, Foundations and Trends in Networking 3 (2) (2009) $127-248$

[39] Y. Shi, Y. T. Hou, J. Liu, S. Kompella, How to correctly use the protocol interference model for multi-hop wireless networks, in: ACM MobiHoc, New Orleans, LA, USA, 2009, pp. 239-248.

[40] H. Huo, Y. Xu, C. C. Bilen, H. Zhang, Coexistence issues of $2.4 \mathrm{ghz}$ sensor networks with other rf devices at home, in: International Conference on Sensor Technologies and Applications (SENSORCOMM), IEEE, 2009, pp. 200-205. doi:10.1109/SENSORCOMM.2009.40

[41] K. Muraoka, T. Watteyne, N. Accettura, X. Vilajosana, K. S. J. Pister, Simple distributed scheduling with collision detection in tsch networks, IEEE Sensors Journal 16 (15) (2016) 5848-5849. doi:10.1109/JSEN.2016.2572961

\section{Appendix A. $K h i^{2}$ test}

To test the independence between the distance and the PDR, we apply the $K h i^{2}$ test. The null hypothesis is that the PDR and the distance are independent. We compute the chi-square indicator by calculating the 
table of theoretical numbers and the discrepancies independence table.

We present here the following values:

1. Table of values (Tab. A.4a);

2. Table of independence (Tab. A.4b;

3. $R^{2}=\left(T-T_{0}\right)^{2}$ (Tab. A.4c);

4. the discrepancies independence table $R^{2} / T_{0}$ (Tab. A.4d);

The chi-square indicator is

$$
\chi^{2}=\sum \frac{R^{2}}{T_{0}}
$$

So, $\chi^{2}=33.38$.

Comparing our indicator with that of the chi-square table, $33.38>18.5$. We reject consequently the hypothesis of independence at the threshold of 0,995 . 